\title{
Quasilinear asymptotically periodic Schrödinger-Poisson system with subcritical growth
}

Jing Zhang ${ }^{1}$, Lifeng Guo ${ }^{2}$ and Miaomiao Yang ${ }^{3 *}$

\author{
Correspondence: 3d07@163.com \\ ${ }^{3}$ School of Mathematics and \\ Statistics, Qilu University of \\ Technology (Shandong Academy of \\ Sciences), Jinan, P.R. China \\ Full list of author information is \\ available at the end of the article
}

\begin{abstract}
The aim of this paper is establishing the existence of a nontrivial solution for the following quasilinear Schrödinger-Poisson system:

$$
\left\{\begin{array}{l}
-\Delta u+V(x) u-u \Delta\left(u^{2}\right)+K(x) \phi(x) u=g(x, u), \quad x \in \mathbb{R}^{3}, \\
-\Delta \phi=K(x) u^{2}, \quad x \in \mathbb{R}^{3}, \\
u \in H^{1}\left(\mathbb{R}^{3}\right), \quad u>0,
\end{array}\right.
$$

where $V, K, g$ are continuous functions. To overcome the technical difficulties caused by the quasilinear term, we change the variable to guarantee the feasibility of applying the mountain pass theorem to solve the above problems. We use the mountain pass theorem and the concentration-compactness principle as basic tools to gain a nontrivial solution the system possesses under an asymptotic periodicity condition at infinity.
\end{abstract}

MSC: $35 \mathrm{~A} 15 ; 35 \mathrm{~B} 33 ; 35 \mathrm{~B} 38$

Keywords: Quasilinear Schrödinger equation; Asymptotically periodic; Mountain pass theorem

\section{Introduction and main results}

In this paper, we consider the following quasilinear asymptotically periodic SchrödingerPoisson system:

$$
\left\{\begin{array}{l}
-\Delta u+V(x) u-u \Delta\left(u^{2}\right)+K(x) \phi(x) u=g(x, u), \quad x \in \mathbb{R}^{3}, \\
-\Delta \phi=K(x) u^{2}, \quad x \in \mathbb{R}^{3}, \\
u \in H^{1}\left(\mathbb{R}^{3}\right), \quad u>0,
\end{array}\right.
$$

where $V, K: \mathbb{R}^{3} \rightarrow \mathbb{R}$ and $g: \mathbb{R}^{3} \times \mathbb{R} \rightarrow \mathbb{R}$ are continuous functions.

The so-called quasilinear Schrödinger-Poisson system was introduced in $[5,25]$ and is a quantum mechanical model of extremely small devices in semiconductor nanostructures taking into account the quantum structure and longitudinal field oscillations during the beam propagation. As far as we know, this new significant physical system was not

c) The Author(s) 2020. This article is licensed under a Creative Commons Attribution 4.0 International License, which permits use, sharing, adaptation, distribution and reproduction in any medium or format, as long as you give appropriate credit to the original author(s) and the source, provide a link to the Creative Commons licence, and indicate if changes were made. The images or other third party material in this article are included in the article's Creative Commons licence, unless indicated otherwise in a credit line to the material. If material is not included in the article's Creative Commons licence and your intended use is not permitted by statutory regulation or exceeds the permitted use, you will need to obtain permission directly from the copyright holder. To view a copy of this licence, visit http://creativecommons.org/licenses/by/4.0/. 
mentioned before [5, 25], even though it is worth exploring from a mathematical point of view.

System (1.1) without quasilinear potential $-u \Delta\left(u^{2}\right)$ is called the Schrödinger-Poisson system,

$$
\left\{\begin{array}{l}
-\Delta u+V(x) u+K(x) \phi(x) u=g(x, u), \quad x \in \mathbb{R}^{3} \\
-\Delta \phi=K(x) u^{2}, \quad x \in \mathbb{R}^{3}
\end{array}\right.
$$

which has been widely studied, and many meaningful results were achieved for the subcritical growth [7, 27, 44, 45, 47] or the critical exponent [46] under various assumptions on the potentials and nonlinearities. Furthermore, nontrivial solutions, radial solutions $[10,13,29]$, ground state solutions [2, 4] and also semiclassical solutions [14, 23, 24] were obtained generally. Moreover, quasilinear Schrödinger equations with $-u \Delta\left(u^{2}\right)$ :

$$
-\Delta u+V(x) u-u \Delta\left(u^{2}\right)=g(x, u), \quad x \in \mathbb{R}^{N},
$$

have been accepted as models of several physical phenomena; we refer the reader to the introduction in [30] and references therein for a discussion on the subject. The existence of solutions of quasilinear equations was considered in $[1,6,11,12,18,43]$.

There are scarce existing results on system (1.1), which is a new combination of two different equations. So our discussion is worth considering and has an excellent prospect; this paper is also an innovation of the pioneering work. When $K(x)=1$ in system (1.1), the authors in [8] took into account the systems by applying the perturbation method, and hence the existence of a sign-changing solution with precisely two nodal domains was derived. The authors in [15] studied the existence and asymptotic behavior of ground state in the whole space $\mathbb{R}^{3}$ for the quasilinear Schrödinger-Poisson system with asymptotically linear $f(t)$ with respect to $t$ at infinity; see also [28] for more related results. Figueiredo and Siciliano $[19,20]$ paid close attention to two different systems with critical growth and obtained the existence and asymptotic behavior of solutions for the previous system in $\mathbb{R}^{3}$ and also achieved a similar result for the latter system in a bounded domain in $\mathbb{R}^{2}$. The authors in [31] considered a system with radial potentials and discontinuous nonlinearity and obtained multiplicity results for radial solutions. By using Ekeland's variational principle and the mountain pass theorem the authors in [33] obtained the existence of the ground state solution for a generalized quasilinear Schrödinger-Poisson system in $\mathbb{R}^{3}$. For related results on nonlinear problems based on variational methods, we refer to [21, 28, 32, 38-42], which are recent contributions to Kirchhoff-type problems. Tang [35] developed a direct method, a non-Nehari manifold method, to find a minimizing Cerami sequence for the energy functional outside the Nehari-Pankov manifold by using the diagonal method. Tang, Lin, and Yu [36] first introduced a local superquadratic condition instead of the classical global superquadratic condition. Chen and Tang [9] proposed a new approach to recover the compactness for the Cerami sequence.

Guided by the method of [34], our main goal is establishing the existence of a solution to problem (1.1) under an asymptotic periodicity condition at infinity.

Letting $\mathcal{F}$ denote the class of functions $h \in C\left(\mathbb{R}^{3}, \mathbb{R}\right) \cap L^{\infty}\left(\mathbb{R}^{3}\right)$ such that, for every $\varepsilon>0$, the set $\left\{x \in \mathbb{R}^{3}:|h(x)| \geq \varepsilon\right\}$ has finite Lebesgue measure, we suppose that $V$ and $K$ are perturbations of periodic functions at infinity. More specifically, we impose the following assumptions on $V$ and $K$ : 
$(V)$ there exist a constant $\alpha>0$ and a function $V_{0} \in C\left(\mathbb{R}^{3}, \mathbb{R}\right), 1$-periodic in $x_{i}, 1 \leq i \leq 3$, such that $V_{0}-V \in \mathcal{F}$ and

$$
V_{0}(x) \geq V(x) \geq \alpha>0, \quad \forall x \in \mathbb{R}^{3} .
$$

(K) $K \in L^{2}\left(\mathbb{R}^{3}\right) \cap L^{\infty}\left(\mathbb{R}^{3}\right)$, and there exist a constant $k>0$ and a function $K_{0} \in C\left(\mathbb{R}^{3}, \mathbb{R}\right) \cap$ $L^{2}\left(\mathbb{R}^{3}\right), 1$-periodic in $x_{i}, 1 \leq i \leq 3$, such that $K_{0}-K \in \mathcal{F}$ and

$$
K_{0}(x) \geq K(x) \geq k>0, \quad \forall x \in \mathbb{R}^{3} .
$$

For $g(x, s)$ and $G(x, s)=\int_{0}^{s} g(x, t) d t$, we need the following hypotheses:

$\left(G_{1}\right) g(x, s)=o(|s|)$ as $s \rightarrow 0^{+}$, uniformly in $\mathbb{R}^{3}$;

$\left(G_{2}\right)$ there are constants $a_{1}, a_{2}>0$ and $8 \leq q_{1}<12$ such that

$$
|g(x, s)| \leq a_{1}+a_{2}|s|^{q_{1}-1}, \quad \forall(x, s) \in \mathbb{R}^{3} \times[0,+\infty)
$$

$\left(G_{3}\right)$ there exist a constant $q_{2}>\frac{3\left(q_{1}-4\right)}{2}$ and a function $h_{1} \in L^{1}\left(\mathbb{R}^{3}\right)$ such that

$$
\frac{1}{4} g(x, s) s-G(x, s) \geq s^{q_{2}}-h_{1}(x), \quad \forall(x, s) \in \mathbb{R}^{3} \times[0,+\infty),
$$

where $q_{1}$ is given by hypothesis $\left(G_{2}\right)$.

We observe that conditions $\left(G_{1}\right)$ and $\left(G_{2}\right)$ allow us to employ variational methods to study problem (1.1) and to verify that the associated functional has a local minimum at the origin. As a matter of fact, we study the functional associated with the modified problem. Condition $\left(G_{2}\right)$ imposes a subcritical growth on $g$. However, under these hypotheses, this functional does not satisfy a compactness condition of Palais-Smale type since the domain is the whole space $\mathbb{R}^{3}$. We also observe that from $\left(G_{2}\right)$ and $\left(G_{3}\right)$ we obtain $0<q_{2} \leq q_{1}$. Note that $22^{*}=\frac{4 N}{N-2}=12$ behaves like a critical exponent for problem (1.1). The asymptotic periodicity of $g$ at infinity is given by the following condition:

$\left(G_{4}\right)$ there exist a constant $4 \leq q_{3}<12$ and functions $h_{2} \in \mathcal{F}$, and $g_{0} \in C\left(\mathbb{R}^{3} \times \mathbb{R}, \mathbb{R}^{+}\right)$, 1 -periodic in $x_{i}, 1 \leq i \leq 3$, such that

(i) $G(x, s) \geq G_{0}(x, s)=\int_{0}^{s} g_{0}(x, t) d t, \forall(x, s) \in \mathbb{R}^{3} \times[0,+\infty)$,

(ii) $\left|g(x, s)-g_{0}(x, s)\right| \leq h_{2}(x) s^{q_{3}-1}, \forall(x, s) \in \mathbb{R}^{3} \times[0,+\infty)$,

(iii) the function $s \rightarrow \frac{g_{0}(x, s)}{s^{7}}$ is nondecreasing in the variable $s>0$.

Finally, we also suppose that $G$ satisfies:

(G) $\frac{G(x, s)}{s^{8}} \rightarrow \infty$ as $s \rightarrow \infty$ uniformly in $\mathbb{R}^{3}$.

The first main result is stated as follows.

Theorem 1.1 Suppose that $(V),(K),\left(G_{1}\right)-\left(G_{4}\right)$, and $(G)$ are satisfied. Then system (1.1) admits a solution.

We observe that in the particular case $V=V_{0}, K=K_{0}$, and $g=g_{0}$, Theorem 1.1 clearly gives us a solution for the periodic problem. Indeed, to show the existence of a solution for the periodic problem, condition $\left(G_{4}\right)$ is not necessary. Consider the following problem:

$$
\left\{\begin{array}{l}
-\Delta u+V_{0}(x) u-u \Delta\left(u^{2}\right)+K_{0}(x) \phi(x) u=g_{0}(x, u), \quad x \in \mathbb{R}^{3}, \\
-\Delta \phi=K_{0}(x) u^{2}, \quad x \in \mathbb{R}^{3}, \\
u \in H^{1}\left(\mathbb{R}^{3}\right), \quad u>0
\end{array}\right.
$$


under the following hypotheses:

$\left(V_{0}\right) \quad V_{0} \in C\left(\mathbb{R}^{3}, \mathbb{R}\right), 1$-periodic in $x_{i}, 1 \leq i \leq 3$, and there exists a constant $\alpha>0$ such that

$$
V_{0}(x) \geq \alpha>0, \quad \forall x \in \mathbb{R}^{3} .
$$

$\left(K_{0}\right) K_{0} \in L^{2}\left(\mathbb{R}^{3}\right) \cap L^{\infty}\left(\mathbb{R}^{3}\right) \cap C\left(\mathbb{R}^{3}\right)$, 1-periodic in $x_{i}, 1 \leq i \leq 3$, and there exists a constant $k>0$ such that

$$
K_{0}(x) \geq k>0, \quad \forall x \in \mathbb{R}^{3} .
$$

Then with the function $g_{0}$ satisfying $\left(G_{1}\right)-\left(G_{3}\right)$ and $(G)$, we obtain the second main result.

Theorem 1.2 Suppose that $\left(V_{0}\right),\left(K_{0}\right),\left(G_{1}\right)-\left(G_{3}\right)$, and $(G)$ are satisfied. Then system (1.2) admits a solution.

The remainder of this paper is organized as follows. In Sect. 2, we present two versions of the mountain pass theorem to be employed later. In Sect. 3, we introduce the variational framework associated with problem (1.1). In Sect. 4, we verify the geometric conditions of the mountain pass theorem and show the boundedness of the Cerami sequences associated with the minimax level. In Sect. 5, we prove the main results, and so the existence of a solution for problems (1.1) and (1.2) is established.

\section{Preliminary}

In this section, we present two versions of the mountain pass theorem due to Ambrosetti and Rabinowitz [3], which are essential tools in this paper.

Let $E$ be a real Banach space, and let $I: E \rightarrow \mathbb{R}$ a functional of class $C^{1}$. Let $M$ be the set of critical points of $I$. For $b \in \mathbb{R}$, we define $I^{b}=\{u \in E: I \leq b\}$ and $M_{b}=\{u \in E$ : $u \in M, I(u)=b\}$.

As we noted in the Introduction, the functional associated with problem (1.1) does not satisfy the Palais-Smale condition. To overcome this difficulty, we will use versions of the mountain pass theorem. To this end, we state the first version of this theorem (see [17, 37]).

We recall that $I \in C^{1}(E, \mathbb{R})$ satisfies the Cerami condition on level $c$, denoted by $(C)_{c}$, if any sequence $\left\{u_{n}\right\} \subset E$ satisfying

$$
\text { (i) } I\left(u_{n}\right) \rightarrow c \text { and (ii) }\left\|I^{\prime}\left(u_{n}\right)\right\|\left(1+\left\|u_{n}\right\|\right) \rightarrow 0
$$

as $n \rightarrow \infty$, possesses a convergent subsequence, where $\|\cdot\|_{E}$ denotes the norm on $E$. A function $I$ satisfies the Cerami condition, denoted by $(C)$, if it satisfies $(C)_{c}$ for every $c \in \mathbb{R}$. We say that $\left\{u_{n}\right\} \subset E$ is a $(C)_{c}$ sequence if it satisfies (i)-(ii).

Theorem 2.1 Let $E$ be a real Banach space, and let $I \in C^{1}(E, \mathbb{R})$. Let $S$ be a closed subset of $E$ that disconnects $E$ into distinct connected components $E_{1}$ and $E_{2}$. Suppose further that $I(0)=0$ and:

(I) $0 \in E_{1}$ and there is $\sigma>0$ such that $\inf _{u \in S} I(u) \geq \sigma>0$,

$\left(I_{2}\right)$ there is $e \in E_{2}$ such that $I(e) \leq 0$. 
Then I possesses $a(C)_{c}$ sequence with $c \geq \sigma>0$ given by

$$
c=\inf _{\gamma \in \Gamma} \max _{t \in[0,1]} I(\gamma(t))
$$

where

$$
\Gamma=\left\{\gamma \in C([0,1], E): \gamma(0)=0, \gamma(1) \in I^{0} \cap E_{2}\right\} .
$$

We will also need a local version of Theorem 2.1, which is proved in [26].

Theorem 2.2 Let $E$ be a real Banach space. Suppose that $I \in C^{1}(E, \mathbb{R})$ satisfies $I(0)=0$, $\left(I_{1}\right)$, and $\left(I_{2}\right)$. If there exists $\gamma_{0} \in \Gamma$, where $\Gamma$ is defined by (2.2), such that

$$
c=\max _{t \in[0,1]} I\left(\gamma_{0}(t)\right)>0,
$$

then I possesses a nontrivial critical point $u \in M_{c} \cap \gamma_{0}([0,1])$.

\section{The variational framework}

In this section, we introduce the variational framework associated with problem (1.1). Here, we consider the space $H^{1}\left(\mathbb{R}^{3}\right)$ endowed with one of the following norms:

$$
\begin{aligned}
& \|u\|=\left(\int_{\mathbb{R}^{3}}\left(|\nabla u|^{2}+V(x) u^{2}\right) d x\right)^{\frac{1}{2}}, \\
& \|u\|_{0}=\left(\int_{\mathbb{R}^{3}}\left(|\nabla u|^{2}+V_{0}(x) u^{2}\right) d x\right)^{\frac{1}{2}} .
\end{aligned}
$$

Note that, in view of condition $(V)$, these norms are both equivalent to the standard norm on $H^{1}\left(\mathbb{R}^{3}\right)$. As far as we know, system (1.1) can be easily transformed into a single nonlinear Schrödinger equation with nonlocal term. Briefly, the Poisson equation is solved by using the Lax-Milgram theorem, so for all $u \in H^{1}\left(\mathbb{R}^{3}\right)$, there is a unique $\phi_{u} \in D^{1,2}\left(\mathbb{R}^{3}\right)$ such that $-\Delta \phi=K(x) u^{2}$, which inserted into the first equation gives

$$
-\Delta u+V(x) u-u \Delta\left(u^{2}\right)+K(x) \phi_{u} u=g(x, u) .
$$

In fact, for each $u \in H^{1}\left(\mathbb{R}^{3}\right)$, we define the operator $T_{u}$ on $D^{1,2}\left(\mathbb{R}^{3}\right)$ by

$$
T_{u}(v)=\int_{\mathbb{R}^{3}} K(x) u^{2} v d x .
$$

The Hölder inequality and the fact $K \in L^{2}\left(\mathbb{R}^{3}\right)$ yield that there is a constant $C>0$ such that for every $v \in D^{1,2}\left(\mathbb{R}^{3}\right)$,

$$
\left|T_{u}(v)\right| \leq C|K|_{2}\|u\|^{2}\|v\|_{D^{1,2}\left(\mathbb{R}^{3}\right)} .
$$

Hence by the Riesz representation theorem there exists a unique $\phi_{u} \in D^{1,2}\left(\mathbb{R}^{3}\right)$ such that

$$
\int_{\mathbb{R}^{3}} \nabla \phi_{u} \nabla v d x=\int_{\mathbb{R}^{3}} K(x) u^{2} v d x
$$


Thus $\phi_{u}$ is a weak solution of $-\Delta \phi_{u}=K(x) u^{2}$ and can be represented by

$$
\phi_{u}(x)=\frac{1}{4 \pi} \int_{\mathbb{R}^{3}} \frac{K(y)}{|x-y|} u^{2}(y) d y .
$$

Moreover, it is obvious that

$$
\|\phi\|_{D^{1,2}\left(\mathbb{R}^{3}\right)}=\left\|T_{u}\right\|_{\mathcal{L}\left(D^{1,2}, \mathbb{R}\right)} \leq C|K|_{2}\|u\|^{2},
$$

where $\mathcal{L}\left(D^{1,2}, \mathbb{R}\right)$ denotes the linear space of linear function from $D^{1,2}$ to $\mathbb{R}$. Also, we have

$$
\int_{\mathbb{R}^{3}} K(x) \phi_{u}(x) u^{2} d x \leq C|K|_{2}\|u\|^{2}\|\phi\|_{D^{1,2\left(\mathbb{R}^{3}\right)}} \leq C|K|_{2}^{2}\|u\|^{4} .
$$

Moreover,

$$
\begin{aligned}
\left|\phi_{u}(x)\right| & =\frac{1}{4 \pi}\left|\int_{B_{1}(0)} \frac{K(y)}{|x-y|} u^{2}(y) d y+\int_{B_{1}^{c}(0)} \frac{K(y)}{|x-y|} u^{2}(y) d y\right| \\
& \leq \frac{1}{4 \pi}\left(|K|_{\infty}\left(\int_{B_{1}(0)} \frac{1}{|x-y|^{2}} d y\right)^{\frac{1}{2}}|u|_{4}^{2}+|K|_{4}\left(\int_{B_{1}^{c}(0)} \frac{1}{|x-y|^{4}} d y\right)^{\frac{1}{4}}|u|_{4}^{2}\right) \\
& <C(K)|u|_{4}^{2} .
\end{aligned}
$$

We observe that formally problem (1.1) is the Euler-Lagrange equation associated with the energy functional

$$
J(u)=\frac{1}{2} \int_{\mathbb{R}^{3}}\left(1+2 u^{2}\right)|\nabla u|^{2} d x+\frac{1}{2} \int_{\mathbb{R}^{3}} V(x) u^{2} d x+\frac{1}{4} \int_{\mathbb{R}^{3}} K(x) \phi_{u} u^{2} d x-\int_{\mathbb{R}^{3}} G(x, u) d x .
$$

From the variational point of view, the first difficulty associated with problem (1.1) is finding an appropriate function space where the functional $J$ is well defined. To avoid such a difficulty, we use the change of variable introduced in [30], that is, we consider $v=f^{-1}(u)$, where $f$ is defined by

$$
\begin{cases}f^{\prime}(t)=\frac{1}{\left(1+2 f^{2}(t)\right)^{\frac{1}{2}}} & \text { on }[0,+\infty), \\ f(t)=-f(-t) & \text { on }[0,+\infty)\end{cases}
$$

having the following properties proved in $[11,16]$.

Lemma 3.1 The function $f$ satisfies the following properties:

(1) $f$ is uniquely defined, $C^{1}$, and invertible;

(2) $\left|f^{\prime}(t)\right| \leq 1$ for all $t \in \mathbb{R}$;

(3) $|f(t)| \leq t$ for all $t \in \mathbb{R}$;

(4) $\frac{f(t)}{t} \rightarrow 1$ as $t \rightarrow 0$;

(5) $\frac{f(t)}{\sqrt{t}} \rightarrow 2^{\frac{1}{4}}$ as $t \rightarrow+\infty$;

(6) $\frac{f(t)}{2} \leq t f^{\prime}(t) \leq f(t)$ for all $t \geq 0$;

(7) $|f(t)| \leq 2^{\frac{1}{4}}|t|^{\frac{1}{2}}$ for all $t \in \mathbb{R}$;

(8) $f^{2}(t)-f(t) f^{\prime}(t) t \geq 0$ for all $t \in \mathbb{R}$; 
(9) there exists a positive constant $C$ such that

$$
|f(t)| \geq \begin{cases}C|t|, & |t| \leq 1 \\ C|t|^{\frac{1}{2}}, & |t| \geq 1\end{cases}
$$

(10) $\left|f(t) f^{\prime}(t)\right| \leq \frac{1}{\sqrt{2}}$ for all $t \in \mathbb{R}$.

As a consequence of Lemma 3.1, the following conclusion is proved in [17].

\section{Corollary 3.1}

(1) The function $f(t) f^{\prime}(t) t^{-1}$ is decreasing for all $t>0$.

(2) The function $f^{3}(t) f^{\prime}(t) t^{-1}$ is increasing for all $t>0$.

So, after the change of variables from $J$, we obtain the following functional:

$$
\begin{aligned}
I(v)= & \frac{1}{2} \int_{\mathbb{R}^{3}}|\nabla v|^{2} d x+\frac{1}{2} \int_{\mathbb{R}^{3}} V(x) f^{2}(v) d x \\
& +\frac{1}{4} \int_{\mathbb{R}^{3}} K(x) \phi_{f(v)} f^{2}(v) d x-\int_{\mathbb{R}^{3}} G(x, f(v)) d x,
\end{aligned}
$$

which is well defined in $H^{1}\left(\mathbb{R}^{3}\right)$ and belongs to $C^{1}$ under the hypotheses $(V),(K),\left(G_{1}\right)$, and $\left(G_{2}\right)$.

Let $F(v)=\int_{\mathbb{R}^{3}} K(x) \phi_{f(v)} f^{2}(v) d x$. Then by Lemma 3.1(3) we have

$$
F(v) \leq \int_{\mathbb{R}^{3}} K(x) \phi_{v} v^{2} \leq C|K|_{2}^{2}\|v\|^{4}
$$

for $v \in H^{1}\left(\mathbb{R}^{3}\right)$. Then by the Lebesgue and Fubini theorems

$$
\begin{aligned}
\lim _{t \rightarrow 0} & \frac{F(v+t w)-F(v)}{t} \\
= & \lim _{t \rightarrow 0}\left(\int_{\mathbb{R}^{3}} \frac{K(x)}{t} \phi_{f(v+t w)} f^{2}(v+t w) d x-\int_{\mathbb{R}^{3}} \frac{K(x)}{t} \phi_{f(v)} f^{2}(v) d x\right) \\
= & \lim _{t \rightarrow 0}\left(\int_{\mathbb{R}^{3}} \frac{K(x)}{t} \frac{1}{4 \pi} \int_{\mathbb{R}^{3}} \frac{K(y)}{|x-y|} f^{2}(v+t w) d y f^{2}(v+t w) d x-\int_{\mathbb{R}^{3}} \frac{K(x)}{t} \phi_{f(v)} f^{2}(v) d x\right) \\
= & \lim _{t \rightarrow 0} \int_{\mathbb{R}^{3}} \frac{K(x)}{t} \frac{1}{4 \pi} \int_{\mathbb{R}^{3}} \frac{K(y)}{|x-y|}\left(f(v)+f^{\prime}(v) t w+o(t)\right)^{2} d y\left(f(v)+f^{\prime}(v) t w+o(t)\right)^{2} d x \\
& -\lim _{t \rightarrow 0} \int_{\mathbb{R}^{3}} \frac{K(x)}{t} \phi_{f(v)} f^{2}(v) d x \\
= & \lim _{t \rightarrow 0}\left(\int_{\mathbb{R}^{3}} \frac{K(x)}{t} \frac{1}{4 \pi} \int_{\mathbb{R}^{3}} \frac{K(y)}{|x-y|} f^{2}(v) d y f^{2}(v) d x\right. \\
& +\int_{\mathbb{R}^{3}} \frac{K(x)}{t} \frac{1}{4 \pi} \int_{\mathbb{R}^{3}} \frac{K(y)}{|x-y|} f^{2}(v) d y 2 f(v) f^{\prime}(v) t w d x \\
& \left.+\int_{\mathbb{R}^{3}} \frac{K(x)}{t} \frac{1}{4 \pi} \int_{\mathbb{R}^{3}} \frac{K(y)}{|x-y|} 2 f(v) f^{\prime}(v) t w d y f^{2}(v) d x\right)+o(t) \\
& -\lim _{t \rightarrow 0} \int_{\mathbb{R}^{3}} \frac{K(x)}{t} \phi_{f(v)} f^{2}(v) d x
\end{aligned}
$$




$$
\begin{aligned}
& =4 \lim _{t \rightarrow 0} \int_{\mathbb{R}^{3}} \frac{1}{4 \pi} \int_{\mathbb{R}^{3}} \frac{K(x) K(y)}{|x-y|} f^{2}(v) f(v) f^{\prime}(v) w d y d x \\
& =4 \int_{\mathbb{R}^{3}} K(x) \phi_{f(v)} f(v) f^{\prime}(v) w d x .
\end{aligned}
$$

As a consequence, the critical points of $I$ are weak solutions of the problem

$$
-\Delta v+V(x) f(v) f^{\prime}(v)+K(x) \phi_{f(v)} f(v) f^{\prime}(v)=g(x, f(v)) f^{\prime}(v), \quad v \in H^{1}\left(\mathbb{R}^{3}\right)
$$

that is,

$$
\begin{aligned}
\langle I(v), w\rangle= & \int_{\mathbb{R}^{3}}\left(\nabla v \nabla w+V(x) f(v) f^{\prime}(v) w\right) d x \\
& +\int_{\mathbb{R}^{3}} K(x) \phi_{f(v)} f(v) f^{\prime}(v) w d x-\int_{\mathbb{R}^{3}} g(x, f(v)) f^{\prime}(v) w d x \\
= & 0
\end{aligned}
$$

for all $v, w \in H^{1}\left(\mathbb{R}^{3}\right)$. We observe (see $\left.[11,16]\right)$ that if $v \in C^{2}\left(\mathbb{R}^{3}\right) \cap H^{1}\left(\mathbb{R}^{3}\right)$ is a critical point of the functional $I$, then the function $u=f(v)$ is a classical solution of problem (1.1).

Remark 3.1 We also observe that to obtain a nonnegative solution for (3.4), we set $g(x, s)=$ 0 for all $x \in \mathbb{R}^{3}, s<0$. Indeed, let $v$ be a critical point of $I$. Taking $w=-v^{-}$, where $v^{-}=$ $\max \{-v, 0\}$, we get

$$
\int_{\mathbb{R}^{3}}\left(\left|\nabla v^{-}\right|^{2}+V(x) f(v) f^{\prime}(v)\left(-v^{-}\right) d x+\int_{\mathbb{R}^{3}} K(x) \phi_{f(v)} f(v) f^{\prime}(v)\left(-v^{-}\right) d x=0\right.
$$

Since $f(v)\left(-v^{-}\right) \geq 0$, we have

$$
\int_{\mathbb{R}^{3}}\left|\nabla v^{-}\right|^{2} d x=0, \quad \int_{\mathbb{R}^{3}} \frac{V(x) f(v)\left(-v^{-}\right)}{\sqrt{1+2 f^{2}(v)}} d x=0,
$$

and

$$
\int_{\mathbb{R}^{3}} \frac{K(x) \phi_{f(v)} f(v)\left(-v^{-}\right)}{\sqrt{1+2 f^{2}(v)}} d x=0
$$

Hence we may conclude that $v^{-}=0$ almost everywhere in $\mathbb{R}^{3}$ and, therefore, $v=v^{+} \geq 0$. As $u=f(v)$, we conclude that $u$ is a nonnegative solution for problem (1.1).

Similarly, associated with the periodic problem, we have the functional $I_{0} \in$ $C^{1}\left(H^{1}\left(\mathbb{R}^{3}\right), \mathbb{R}\right)$ defined by

$$
\begin{aligned}
I_{0}(v)= & \frac{1}{2} \int_{\mathbb{R}^{3}}|\nabla v|^{2} d x+\frac{1}{2} \int_{\mathbb{R}^{3}} V_{0}(x) f^{2}(v) d x \\
& +\frac{1}{4} \int_{\mathbb{R}^{3}} K_{0}(x) \widetilde{\phi}_{f(v)} f^{2}(v) d x-\int_{\mathbb{R}^{3}} G_{0}(x, f(v)) d x
\end{aligned}
$$

where $G_{0}(x, s)=0$ for all $x \in \mathbb{R}^{3}, s<0$. 
We also observe that for $\delta>0$, because of $\left(G_{1}\right)$ and $\left(G_{2}\right)$, there is a constant $C_{\delta}>0$ such that

$$
\begin{aligned}
& |g(x, s)| \leq \delta|s|+C_{\delta}|s|^{q_{1}-1}, \quad \forall(x, s) \in \mathbb{R}^{3} \times \mathbb{R}, \\
& |G(x, s)| \leq \frac{\delta}{2}|s|^{2}+\frac{C_{\delta}}{q_{1}}|s|^{q_{1}}, \quad \forall(x, s) \in \mathbb{R}^{3} \times \mathbb{R} .
\end{aligned}
$$

\section{Geometric properties}

In this section, we present the variational results that will be used in the proof of main results. Firstly, we verify the geometric conditions of the mountain pass theorem. Then we present results concerning the behavior of the Cerami sequences of the associated functional. Finally, we establish two technical results necessary for the proof of Theorem 1.1. The following lemma shows that the (modified) functional associated with problem (1.1) satisfies the geometric properties of the mountain pass theorem.

Lemma 4.1 Suppose that $(V),\left(G_{1}\right),\left(G_{2}\right)$, and $(G)$ are satisfied. Then the functional I defined by (3.3) satisfies conditions $\left(I_{1}\right)$ and $\left(I_{2}\right)$ of Theorem 2.1 .

Proof For $\rho>0$, define

$$
S_{\rho}=\left\{v \in H^{1}\left(\mathbb{R}^{3}\right): \int_{\mathbb{R}^{3}}\left(|\nabla v|^{2}+V(x) f^{2}(v)\right) d x=\rho^{2}\right\} .
$$

Since $\Psi: H^{1}\left(\mathbb{R}^{3}\right) \rightarrow \mathbb{R}$ given by

$$
\Psi(v)=\int_{\mathbb{R}^{3}}\left(|\nabla v|^{2}+V(x) f^{2}(v)\right) d x
$$

is continuous, $S_{\rho}$ is a closed subset that disconnects the space $H^{1}\left(\mathbb{R}^{3}\right) \rightarrow \mathbb{R}$. Taking $0<$ $\lambda<1$ such that $\frac{q_{1}}{2}=\lambda+6(1-\lambda)$, by $(V)$, relation (3.7), Hölder's inequality, Lemma 3.1(7), the Sobolev embedding theorem, and the result of [34] we have

$$
\int_{\mathbb{R}^{3}} G(x, f(v)) d x \leq \frac{\delta}{2 \alpha} \rho^{2}+\frac{2^{3(1-\lambda)} C_{0} C_{\delta}}{q_{1} \alpha^{\lambda}} \rho^{2 \lambda+6(1-\lambda)}
$$

for every $v \in S_{\rho}$ and some constant $C_{0}>0$. Hence we have

$$
I(v) \geq\left(\frac{1}{2}-\frac{\delta}{2 \alpha}\right) \rho^{2}-\frac{2^{3(1-\lambda)} C_{0} C_{\delta}}{q_{1} \alpha^{\lambda}} \rho^{2 \lambda+6(1-\lambda)}
$$

for every $v \in S_{\rho}$. Since $2 \lambda+6(1-\lambda)>2$, choosing $0<\delta<\alpha$, we conclude that, for $\rho$ sufficiently small,

$$
c_{0}:=\inf _{S_{\rho}} I \geq \sigma>0
$$

that is, condition $\left(I_{1}\right)$ is satisfied.

To verify condition $\left(I_{2}\right)$, it suffices to exhibit $w \in H^{1}\left(\mathbb{R}^{3}\right)$ such that

$$
I(t w) \rightarrow-\infty \quad \text { as } t \rightarrow+\infty .
$$


Indeed, consider $w \in H^{1}\left(\mathbb{R}^{3}\right)$. Hence by properties (3) of Lemma 3.1 we get, for every $t>0$,

$$
\begin{aligned}
I(t w) \leq & \frac{t^{2}}{2} \int_{\mathbb{R}^{3}}\left(|\nabla w|^{2}+V(x) w^{2}\right) d x+\frac{t^{4}}{4} \int_{\mathbb{R}^{3}} K(x) \phi_{w} w^{2} d x-\int_{\mathbb{R}^{3}} G(x, f(t w)) d x \\
\leq & \frac{t^{2}}{2} \int_{\mathbb{R}^{3}}\left(|\nabla w|^{2}+V(x) w^{2}\right) d x+\frac{t^{4}}{4}|K|_{2}\left|\phi_{w}\right|_{6}|w|_{6}^{2} \\
& -t^{4} \int_{w \neq 0} \frac{G(x, f(t w))}{f^{8}(t w)} \frac{f^{8}(t w)}{(t w)^{4}} w^{4} d x .
\end{aligned}
$$

Then by Lemma 3.1(5), assumption (G), and the Fatou lemma the last integral on the right-hand side tends to infinity with $t$. Hence $I(t w) \rightarrow-\infty$ as $t \rightarrow \infty$.

As a consequence of Theorem 2.1 and Lemma 3.1, we have the following corollary.

Corollary 4.1 Suppose that $(V),\left(G_{1}\right),\left(G_{2}\right)$, and $(G)$ are satisfied. Then the functional I possesses a $(C)_{c}$ sequence with $c$ given by (2.1).

Here we verify the boundedness of the $(C)_{c}$ sequences associated with the functional $I$ and a result concerning the behavior of such sequences.

Lemma 4.2 Suppose that $(V)$ and $\left(G_{1}\right)-\left(G_{3}\right)$ are satisfied. Then every Cerami sequence $\left\{v_{n}\right\}$ in $H^{1}\left(\mathbb{R}^{3}\right)$ associated with the functional I is bounded.

Proof According to the proof of [34], if there exists a constant $M>0$ such that

$$
\int_{\mathbb{R}^{3}}\left(\left|\nabla v_{n}\right|^{2}+V(x) f^{2}\left(v_{n}\right)\right) d x \leq M
$$

then $\left\{v_{n}\right\}$ is bounded in $H^{1}\left(\mathbb{R}^{3}\right)$. So we only need to prove (4.2).

Now let $\left\{v_{n}\right\} \in H^{1}\left(\mathbb{R}^{3}\right)$ be an arbitrary Cerami sequence for $I$ on level $c \in \mathbb{R}$, that is,

$$
I\left(v_{n}\right) \rightarrow c, \quad\left\|I^{\prime}\left(v_{n}\right)\right\|\left(1+\left\|v_{n}\right\|\right) \rightarrow 0 .
$$

Taking $0<\delta<\alpha$, by the first condition in (4.3), (3.7), and the condition $(V)$ we have

$$
\begin{aligned}
& \frac{1}{2} \int_{\mathbb{R}^{3}}\left(\left|\nabla v_{n}\right|^{2}+V(x) f^{2}\left(v_{n}\right)\right) d x+\frac{1}{4} \int_{\mathbb{R}^{3}} K(x) \phi_{v_{n}} f^{2}\left(v_{n}\right) d x \\
& \quad=\int_{\mathbb{R}^{3}} G\left(x, f\left(v_{n}\right)\right) d x+c+o_{n}(1) \\
& \left.\quad \leq \frac{\delta}{2 \alpha} \int_{\mathbb{R}^{3}} V(x) f^{2}\left(v_{n}\right)\right) d x+\frac{C_{\delta}}{q_{1}} \int_{\mathbb{R}^{3}}\left|f\left(v_{n}\right)\right|^{q_{1}} d x+c+o_{n}(1),
\end{aligned}
$$

which yields

$$
\begin{aligned}
& \frac{1}{2} \int_{\mathbb{R}^{3}}\left|\nabla v_{n}\right|^{2} d x+\left(\frac{1}{2}-\frac{\delta}{2 \alpha}\right) \int_{\mathbb{R}^{3}} V(x) f^{2}\left(v_{n}\right) d x+\frac{1}{4} \int_{\mathbb{R}^{3}} K(x) \phi_{f\left(v_{n}\right)} f^{2}\left(v_{n}\right) d x \\
& \quad \leq \frac{C_{\delta}}{q_{1}} \int_{\mathbb{R}^{3}}\left|f\left(v_{n}\right)\right|^{q_{1}} d x+c+o_{n}(1) .
\end{aligned}
$$


By the second condition in (4.3) and Lemma 3.1(6) we have

$$
\begin{aligned}
\left\langle I^{\prime}\left(v_{n}\right), v_{n}\right\rangle \leq & \int_{\mathbb{R}^{3}}\left(\left|\nabla v_{n}\right|^{2}+V(x) f^{2}\left(v_{n}\right)\right) d x+\int_{\mathbb{R}^{3}} K(x) \phi_{f\left(v_{n}\right)} f^{2}\left(v_{n}\right) d x \\
& -\int_{\mathbb{R}^{3}} \frac{1}{2} g\left(x, f\left(v_{n}\right)\right) f\left(v_{n}\right) d x .
\end{aligned}
$$

Consequently, by $\left(G_{3}\right)$ we have

$$
\begin{aligned}
I\left(v_{n}\right) & -\frac{1}{2}\left\langle I^{\prime}\left(v_{n}\right), v_{n}\right\rangle \\
& \geq-\frac{1}{4} \int_{\mathbb{R}^{3}} K(x) \phi_{f\left(v_{n}\right)} f^{2}\left(v_{n}\right) d x+\int_{\mathbb{R}^{3}}\left[\frac{1}{4} g\left(x, f\left(v_{n}\right)\right) f\left(v_{n}\right)-G\left(x, f\left(v_{n}\right)\right)\right] d x \\
& \geq-\frac{1}{4} \int_{\mathbb{R}^{3}} K(x) \phi_{f\left(v_{n}\right)} f^{2}\left(v_{n}\right) d x+\int_{\mathbb{R}^{3}}\left|f\left(v_{n}\right)\right|^{q 2} d x-\int_{\mathbb{R}^{3}} h_{1}(x) d x .
\end{aligned}
$$

Since $h_{1} \in L^{1}\left(\mathbb{R}^{3}\right)$, we have

$$
\int_{\mathbb{R}^{3}}\left|f\left(v_{n}\right)\right|^{q 2} d x \leq C+\frac{1}{4} \int_{\mathbb{R}^{3}} K(x) \phi_{f\left(v_{n}\right)} f^{2}\left(v_{n}\right) d x .
$$

Because of Lemma 3.1(3), we have

$$
\int_{\mathbb{R}^{3}} K(x) \phi_{f\left(v_{n}\right)} f^{2}\left(v_{n}\right) d x \leq \int_{\mathbb{R}^{3}} K(x) \phi_{v_{n}} v_{n}^{2} d x \leq|K|_{2}\left|\phi_{v_{n}}\right|{ }_{6}\left|v_{n}\right|_{6}^{2} .
$$

Therefore

$$
\int_{\mathbb{R}^{3}}\left|f\left(v_{n}\right)\right|^{q_{2}} d x \leq C_{1}
$$

As we noted in the Introduction, $q_{2} \leq q_{1}$. If $q_{2}=q_{1}$, then inequality (4.2) is given by (4.4), (4.5), and our choice of $\delta$. Now consider $q_{2}<q_{1}$. Let $0<\lambda<1$ be such that $q_{1}=\lambda q_{2}+$ $12(1-\lambda)$. By Hölder's inequality, (4.4), (4.5), Lemma 3.1(7), and the Sobolev embedding theorem we have

$$
\begin{aligned}
& \frac{1}{2} \int_{\mathbb{R}^{3}}\left|\nabla v_{n}\right|^{2} d x+\left(\frac{1}{2}-\frac{\delta}{2 \alpha}\right) \int_{\mathbb{R}^{3}} V(x) f^{2}\left(v_{n}\right) d x+\frac{1}{4} \int_{\mathbb{R}^{3}} K(x) \phi_{f\left(v_{n}\right)} f^{2}\left(v_{n}\right) d x \\
& \quad \leq \frac{C_{\delta}}{q_{1}}\left(\int_{\mathbb{R}^{3}}\left|f\left(v_{n}\right)\right|^{q_{2}} d x\right)^{\lambda}\left(\int_{\mathbb{R}^{3}}\left|f\left(v_{n}\right)\right|^{12} d x\right)^{1-\lambda}+c+o_{n}(1) \\
& \quad \leq \frac{C_{\delta}}{q_{1}} C_{1}^{\lambda} 2^{3(1-\lambda)}\left(\int_{\mathbb{R}^{3}}\left|v_{n}\right|^{6} d x\right)^{1-\lambda}+c+o_{n}(1) \\
& \quad \leq C \frac{C_{\delta}}{q_{1}} C_{1}^{\lambda} 2^{3(1-\lambda)}\left(\int_{\mathbb{R}^{3}}\left|\nabla v_{n}\right|^{2} d x\right)^{3(1-\lambda)}+c+o_{n}(1) .
\end{aligned}
$$

Now note that by $\left(G_{3}\right)$ that $3(1-\lambda)=\frac{3\left(q_{1}-q_{2}\right)}{12-q_{2}}<1$. Therefore estimate (4.2) is satisfied, and the lemma is proved. 
Lemma 4.3 Suppose that $(V),\left(G_{1}\right)$, and $\left(G_{2}\right)$ are satisfied. Let $\left\{v_{n}\right\} \subset H^{1}\left(\mathbb{R}^{3}\right)$ be a $(C)_{c}$ sequence with c given by (2.1), and let $v_{n} \rightarrow 0$ weakly in $H^{1}\left(\mathbb{R}^{3}\right)$. Then there exist a sequence $\left\{y_{n}\right\} \subset \mathbb{R}^{3}$ and $r, \eta>0$ such that $\left|y_{n}\right| \rightarrow \infty$ and

$$
\lim _{n \rightarrow \infty} \sup \int_{B_{r}\left(y_{n}\right)}\left|v_{n}\right|^{2} d x \geq \eta>0
$$

Proof Suppose on the contrary that

$$
\lim _{n \rightarrow \infty} \sup \int_{B_{r}\left(y_{n}\right)}\left|v_{n}\right|^{2} d x=0, \quad \forall r>0
$$

Then by Lion's concentration compactness lemma (see [37, Lemma 1.21]) we have

$$
u_{n} \rightarrow 0 \text { in } L^{p}\left(\mathbb{R}^{3}\right) \text { for } 2<p<6 .
$$

According to Lemma 3.1(3, 6), we have

$$
\begin{aligned}
& \lim _{n \rightarrow \infty} \sup \int_{\mathbb{R}^{3}} K(x) \phi_{f\left(v_{n}\right)} f\left(v_{n}\right) f^{\prime}\left(v_{n}\right) v_{n} d x \\
& \quad \leq \lim _{n \rightarrow \infty} \sup \int_{\mathbb{R}^{3}} K(x) \phi_{f\left(v_{n}\right.} f^{2}\left(v_{n}\right) d x \\
& \quad \leq \lim _{n \rightarrow \infty} \sup \int_{\mathbb{R}^{3}} K(x) \phi_{v_{n}} v_{n}^{2} d x \leq|K|_{\infty}\left|\phi_{v_{n}}\right|_{6} \lim _{n \rightarrow \infty}\left(\int_{\mathbb{R}^{3}} v_{n}^{\frac{12}{5}} d x\right)^{\frac{5}{6}}=0 .
\end{aligned}
$$

Let $0<\beta<2$ be sufficiently small such that $2+\beta<q_{1}<12-2 \beta$. Now take $0<\lambda<1$ such that $q_{1}=\lambda(2+\beta)+(1-\lambda)(12-2 \beta)$. Applying Hölder's inequality and Lemma 3.1(3, 7), we have

$$
\begin{aligned}
\int_{\mathbb{R}^{3}}\left|f\left(v_{n}\right)\right|^{q_{1}} d x & \leq\left(\int_{\mathbb{R}^{3}}\left|f\left(v_{n}\right)\right|^{2+\beta} d x\right)^{\lambda}\left(\int_{\mathbb{R}^{3}}\left|f\left(v_{n}\right)\right|^{2(6-\beta)} d x\right)^{1-\lambda} \\
& \leq 2^{\frac{(1-\lambda)(6-\beta)}{2}}\left(\int_{\mathbb{R}^{3}}\left|v_{n}\right|^{2+\beta} d x\right)^{\lambda}\left(\int_{\mathbb{R}^{3}}\left|v_{n}\right|^{6-\beta} d x\right)^{1-\lambda} .
\end{aligned}
$$

Noting that $2<2+\beta, 6-\beta<6$, from (3.6), Lemma 3.1(6), and (4.6) it follows that for all $\delta>0$,

$$
\begin{aligned}
& \lim _{n \rightarrow \infty} \sup \int_{\mathbb{R}^{3}}\left|g\left(x, f\left(v_{n}\right)\right) f^{\prime}\left(v_{n}\right) v_{n}\right| d x \\
& \quad \leq \lim _{n \rightarrow \infty} \sup \int_{\mathbb{R}^{3}}\left|g\left(x, f\left(v_{n}\right)\right) f\left(v_{n}\right)\right| d x \\
& \quad \leq \lim _{n \rightarrow \infty} \sup \left[\delta \int_{\mathbb{R}^{3}}\left|v_{n}\right|^{2} d x+C_{\delta} 2^{\frac{(1-\lambda)(6-\beta)}{2}}\left(\int_{\mathbb{R}^{3}}\left|v_{n}\right|^{2+\beta} d x\right)^{\lambda}\left(\int_{\mathbb{R}^{3}}\left|v_{n}\right|^{6-\beta} d x\right)^{1-\lambda}\right] \\
& \quad=\delta \lim _{n \rightarrow \infty} \sup \int_{\mathbb{R}^{3}}\left|v_{n}\right|^{2} d x .
\end{aligned}
$$


Hence, since $\delta>0$ can be chosen arbitrarily small and the sequence $\left\{v_{n}\right\} \subset H^{1}\left(\mathbb{R}^{3}\right)$ is bounded, we get

$$
\lim _{n \rightarrow \infty} \int_{\mathbb{R}^{3}}\left|g\left(x, f\left(v_{n}\right)\right) f^{\prime}\left(v_{n}\right) v_{n}\right| d x=0 .
$$

Since $\left\langle I^{\prime}\left(v_{n}\right), v_{n}\right\rangle \rightarrow 0$, from (4.7) and (4.8) we obtain

$$
\lim _{n \rightarrow \infty} \int_{\mathbb{R}^{3}}\left|\nabla v_{n}\right|^{2}+V(x) f\left(v_{n}\right) f^{\prime}\left(v_{n}\right) v_{n} d x=0 .
$$

Using Lemma 3.1(6), we get

$$
\lim _{n \rightarrow \infty} \int_{\mathbb{R}^{3}}\left(\left|\nabla v_{n}\right|^{2}+V(x) f^{2}\left(v_{n}\right)\right) d x=0 .
$$

By an argument similar to that used to verify (4.7) and (4.8) we conclude that

$$
\lim _{n \rightarrow \infty} \int_{\mathbb{R}^{3}} K(x) \phi_{f\left(v_{n}\right)} f^{2}\left(v_{n}\right) d x=0
$$

and

$$
\lim _{n \rightarrow \infty} \int_{\mathbb{R}^{3}} G\left(x, f\left(v_{n}\right)\right) d x=0 .
$$

These two equalities, together with (4.9), imply that $I\left(v_{n}\right) \rightarrow 0$, which contradicts $I\left(v_{n}\right) \rightarrow$ $c>0$. The lemma is proved.

Lemma 4.4 Suppose that $(V),(K)$, and $\left(G_{4}\right)$ are satisfied. Let $\left\{v_{n}\right\} \subset H^{1}\left(\mathbb{R}^{3}\right)$ be a bounded sequence, and let $w_{n}(x)=w\left(x-y_{n}\right)$, where $w \in H^{1}\left(\mathbb{R}^{3}\right)$ and $\left(y_{n}\right) \subset \mathbb{R}^{3}$. If $\left|y_{n}\right| \rightarrow \infty$, then, as $n \rightarrow \infty$, we have

(1) $\int_{\mathbb{R}^{3}}\left[V_{0}(x)-V(x)\right] f\left(v_{n}\right) f^{\prime}\left(v_{n}\right) w_{n} d x \rightarrow 0$,

(2) $\int_{\mathbb{R}^{3}}\left[g_{0}\left(x, f\left(v_{n}\right)\right)-g\left(x, f\left(v_{n}\right)\right)\right] f^{\prime}\left(v_{n}\right) w_{n} d x \rightarrow 0$,

(3) $\int_{\mathbb{R}^{3}} K_{0}(x) \widetilde{\phi}_{f\left(v_{n}\right)} f\left(v_{n}\right) f^{\prime}\left(v_{n}\right) w_{n} d x-K(x) \phi_{f\left(v_{n}\right)} f\left(v_{n}\right) f^{\prime}\left(v_{n}\right) w_{n} d x \rightarrow 0$.

Proof The proof of (1) and (2) can found in [34], so we only need to prove (3). Let $\zeta(x)=$ $K_{0}(x)-K(x)$. Then by Lemma $3.1(2,3)$ we have

$$
\begin{aligned}
\int_{\mathbb{R}^{3}} \mid & K_{0}(x) \widetilde{\phi}_{f\left(v_{n}\right)} f\left(v_{n}\right) f^{\prime}\left(v_{n}\right) w_{n}-K(x) \phi_{f\left(v_{n}\right)} f\left(v_{n}\right) f^{\prime}\left(v_{n}\right) w_{n} \mid d x \\
\leq & \int_{\mathbb{R}^{3}}\left|K_{0}(x) \widetilde{\phi}_{v_{n}}-K(x) \phi_{v_{n}}\right|\left|v_{n}\right|\left|w_{n}\right| d x \\
= & \frac{1}{4 \pi}\left(\int_{\mathbb{R}^{3}} \int_{\mathbb{R}^{3}} \frac{K(y)}{|x-y|}\left|v_{n}\right|\left|w_{n}\right| d y\left(K_{0}(x)-K(x)\right) v_{n}^{2} d x\right. \\
& +\int_{\mathbb{R}^{3}} \int_{\mathbb{R}^{3}} \frac{K(y)}{|x-y|} v_{n}^{2} d y\left(K_{0}(x)-K(x)\right)\left|v_{n}\right|\left|w_{n}\right| d x \\
& \left.+\int_{\mathbb{R}^{3}} \int_{\mathbb{R}^{3}} \frac{\left(K_{0}(y)-K(y)\right)}{|x-y|} v_{n}^{2} d y\left(K_{0}(x)-K(x)\right)\left|v_{n}\right|\left|w_{n}\right| d x\right) \\
= & I_{1}+I_{2}+I_{3} .
\end{aligned}
$$


We consider, for example, $I_{2}$. Then the other two can be obtained in the same way.

$$
\begin{aligned}
I_{2} & =\int_{B_{R}(0)}\left(K_{0}(x)-K(x)\right) \phi_{v_{n}}\left|v_{n}\right|\left|w_{n}\right| d x+\int_{\mathbb{R}^{3} \backslash\left(B_{R}(0)\right.}\left(K_{0}(x)-K(x)\right) \phi_{v_{n}}\left|v_{n}\right|\left|w_{n}\right| d x \\
& =I_{21}+I_{22} .
\end{aligned}
$$

Given $\delta>0$, since $w \in H^{1}\left(\mathbb{R}^{3}\right) \subset L^{p}\left(\mathbb{R}^{3}\right)$ with $2 \leq p \leq 6$, we can find $0<\varepsilon<\delta$ such that, for every measurable set $\Omega \subset \mathbb{R}^{3}$ satisfying $|\Omega|<\varepsilon$,

$$
\int_{\Omega}|w|^{2} d x<\delta, \quad \int_{\Omega}|w|^{6} d x<\delta
$$

We fix $\varepsilon>0$ and set $D_{\varepsilon}(R)=\left\{x \in \mathbb{R}^{3}:\left|K_{0}(x)-K(x)\right| \geq \varepsilon,|x| \geq R\right\}$. By condition $(K)$ we can find $R>0$ such that $\left|D_{\varepsilon}(R)\right|<\varepsilon$. Then, according to (3.2), applying Lemma 3.1(2,3), Hölder's inequality, condition $(K), h \in \mathcal{F}$, and (4.10), we get

$$
\begin{aligned}
\left|I_{22}\right|= & \left|\phi_{v_{n}}\right|_{\infty}\left(\int_{D_{\varepsilon}(R)}\left(K_{0}(x)-K(x)\right)\left|v_{n}\right|\left|w_{n}\right| d x\right. \\
& \left.+\int_{\mathbb{R}^{3} \backslash\left(B_{R}(0) \cup D_{\varepsilon}(R)\right)}\left(K_{0}(x)-K(x)\right)\left|v_{n}\right|\left|w_{n}\right| d x\right) \\
\leq & \left|\phi_{v_{n}}\right|_{\infty}\left(|h|_{\infty} \int_{D_{\varepsilon}(R)}\left|v_{n}\right|\left|w_{n}\right| d x+\varepsilon \int_{\mathbb{R}^{3} \backslash\left(B_{R}(0) \cup D_{\varepsilon}(R)\right)}\left|v_{n}\right|\left|w_{n}\right| d x\right) \\
\leq & \left|\phi_{v_{n}}\right|_{\infty}\left(|h|_{\infty}\left|v_{n}\right|_{2}\left(\int_{D_{\varepsilon}(R)}\left|w_{n}\right|^{2} d x\right)^{\frac{1}{2}}+\varepsilon\left|v_{n}\right|_{2}\left|w_{n}\right|_{2}\right) \\
\leq & C\left(\delta^{\frac{1}{2}}+\delta\right) .
\end{aligned}
$$

Applying Hölder's inequality, condition $(K)$, Lemma 3.1(2,3), and the fact that $\left\{v_{n}\right\} \subset$ $H^{1}\left(\mathbb{R}^{3}\right)$ is bounded, we can find $\widetilde{C}$ such that

$$
\begin{aligned}
\left|I_{21}\right| & \leq\left|\phi_{v_{n}}\right|_{\infty} \int_{B_{R}(0)}\left|K_{0}(x)-K(x)\right|\left|v_{n}\right|\left|w_{n}\right| d x \\
& \leq\left.\left|\phi_{v_{n}}\right|_{\infty}|h|_{\infty}|| v_{n}\right|_{2}\left(\int_{B_{R}(0)}\left|w_{n}\right|^{2} d x\right)^{\frac{1}{2}} \\
& \leq \widetilde{C}\left(\int_{B_{R}\left(-y_{n}\right)}|w(x)|^{2} d x\right)^{\frac{1}{2}} .
\end{aligned}
$$

Hence, since $w \in H^{1}\left(\mathbb{R}^{3}\right)$ and $\left|y_{n}\right| \rightarrow \infty$, there is $n_{0} \in \mathbb{N}$ such that

$$
\int_{B_{R}(0)}\left(K_{0}(x)-K(x)\right) \phi_{v_{n}}\left|v_{n}\right|\left|w_{n}\right| d x \leq \widetilde{C} \delta, \quad \forall n \geq n_{0} .
$$

Since $\delta>0$ can be chosen arbitrarily small, the last three inequalities imply that

$$
\int_{\mathbb{R}^{3}} K_{0}(x) \widetilde{\phi}_{f\left(v_{n}\right)} f\left(v_{n}\right) f^{\prime}\left(v_{n}\right) w_{n} d x-K(x) \phi_{f\left(v_{n}\right)} f\left(v_{n}\right) f^{\prime}\left(v_{n}\right) w_{n} d x \rightarrow 0
$$

strongly in $L^{1}\left(\mathbb{R}^{3}\right)$ as $n \rightarrow \infty$. 
Lemma 4.5 (see [34]) Suppose that $2 \leq q \leq 12$ and $h \in \mathcal{F}$. Let $\left\{v_{n}\right\} \subset H^{1}\left(\mathbb{R}^{3}\right)$ be a sequence such that $v_{n} \rightarrow v$ weakly in $H^{1}\left(\mathbb{R}^{3}\right)$. Then

$$
\int_{\mathbb{R}^{3}} h(x)\left|f\left(v_{n}\right)\right|^{q} d x \rightarrow \int_{\mathbb{R}^{3}} h(x)|f(v)|^{q} d x
$$

as $n \rightarrow \infty$.

\section{Proofs of the main results}

In this section, we prove Theorems 1.1 and 1.2 by verifying that the functionals $I$ and $I_{0}$, defined by (3.3) and (3.5), respectively, have nontrivial critical points.

Proof of Theorem 1.1 Firstly, by Corollary 4.1 we can find a Cerami sequence $\left\{v_{n}\right\}$ on level $c$, that is, such that

$$
I\left(v_{n}\right) \rightarrow c \geq \sigma>0 \quad \text { and } \quad\left\|I^{\prime}\left(v_{n}\right)\right\|\left(1+\left\|v_{n}\right\|\right) \rightarrow 0
$$

as $n \rightarrow \infty$ with $c$ given by Theorem 2.1. Applying Lemma 4.3, we may assume, without loss of generality, that $v_{n} \rightarrow v$ weakly in $H^{1}\left(\mathbb{R}^{3}\right)$.

Step 1 . Note that, for every $z \in C_{0}^{\infty}\left(\mathbb{R}^{3}\right)$,

$$
\begin{aligned}
& \left\langle I^{\prime}\left(v_{n}\right), z\right\rangle-\left\langle I^{\prime}(v), z\right\rangle \\
& =\int_{\mathbb{R}^{3}} \nabla\left(v_{n}-v\right) \nabla z d x+\int_{\mathbb{R}^{3}} V(x)\left[f\left(v_{n}\right) f^{\prime}\left(v_{n}\right)-f(v) f^{\prime}(v)\right] z d x \\
& \quad+\int_{\mathbb{R}^{3}} K(x)\left[\phi_{f\left(v_{n}\right)} f\left(v_{n}\right) f^{\prime}\left(v_{n}\right)-\phi_{f(v)} f(v) f^{\prime}(v)\right] z d x \\
& \quad-\int_{\mathbb{R}^{3}}\left[g\left(x, f\left(v_{n}\right)\right) f^{\prime}\left(v_{n}\right)-g(x, f(v)) f^{\prime}(v)\right] z d x .
\end{aligned}
$$

Since $v_{n} \rightarrow v$ weakly in $H^{1}\left(\mathbb{R}^{3}\right)$, we have that $v_{n} \rightarrow v$ in $L_{\text {loc }}^{s}\left(\mathbb{R}^{3}\right)$ with $1 \leq s<6$. Then, up to a subsequence,

$$
\begin{aligned}
& v_{n}(x) \rightarrow v(x) \quad \text { a.e. on } \Omega=\operatorname{supp} z \text { as } n \rightarrow \infty, \\
& \left|v_{n}(x)\right| \leq\left|\omega_{s}(x)\right| \text { for every } n \in \mathbb{N} \text { and a.e. on } \Omega \text {, with } \omega_{s} \in L^{s} .
\end{aligned}
$$

Consequently, as $n \rightarrow \infty$,

$$
\begin{aligned}
& f\left(v_{n}\right) f^{\prime}\left(v_{n}\right) \rightarrow f(v) f^{\prime}(v), \\
& \phi_{f\left(v_{n}\right)} f\left(v_{n}\right) f^{\prime}\left(v_{n}\right) \rightarrow \phi_{f(v)} f(v) f^{\prime}(v), \\
& g\left(x, f\left(v_{n}\right)\right) f^{\prime}\left(v_{n}\right)-g(x, f(v)) f^{\prime}(v),
\end{aligned}
$$

a.e. on $\Omega$. Furthermore, from conditions $(V)$ and $(K)$, Lemma 3.1 $(2,3)$, and $(3.2)$ we have

$$
\left|V(x) f\left(v_{n}\right) f^{\prime}\left(v_{n}\right) z\right| \leq\left|V(x) v_{n} z\right| \leq|V|_{\infty}\left|\omega_{2}\right||z|
$$

and

$$
\left|K(x) \phi_{f\left(v_{n}\right)} f\left(v_{n}\right) f^{\prime}\left(v_{n}\right) z\right| \leq\left|K(x) \phi_{v_{n}} v_{n} z\right| \leq|K|_{\infty}\left|\phi_{v_{n}}\right|_{\infty}\left|\omega_{2}\right||z| .
$$


Moreover, by (3.6) and Lemma 3.1(2, 3, 6, 7) we have, for $\left|v_{n}(x)\right| \leq 1$,

$$
\mid g\left(x,\left.f\left(v_{n}\right) f^{\prime}\left(v_{n}\right) z|\leq \delta| v_{n}|| z\left|+C_{\delta}\right| f\left(v_{n}\right)\right|^{q_{1}-1}|z| \leq \delta\left|\omega_{2}\right||z|+C_{\delta}\left|\omega_{2}\right||z| .\right.
$$

On the other hand, if $\left|v_{n}(x)\right|>1$, then

$$
\begin{aligned}
\left|g\left(x, f\left(v_{n}\right)\right) f^{\prime}\left(v_{n}\right) z\right| & \leq \delta\left|v_{n}\right||z|+C_{\delta}\left|f\left(v_{n}\right)\right|^{q_{1}-1}\left|f^{\prime}\left(v_{n}\right)\right||z| \\
& \leq \delta\left|\omega_{2}\right||z|+C_{\delta}\left|f\left(v_{n}\right)\right|^{q_{1}-1} \frac{\left|f\left(v_{n}\right)\right|}{\left|v_{n}\right|}|z| \\
& \leq \delta\left|\omega_{2}\right||z|+2^{3} C_{\delta}\left|\omega_{5}\right|^{5}|z| .
\end{aligned}
$$

The two estimates imply that there exists a function $\psi \in L^{1}(\Omega)$ such that

$$
\mid g\left(x, f\left(v_{n}\right) f^{\prime}\left(v_{n}\right) z \mid \leq \psi \quad \text { in } \mathbb{R}^{3} .\right.
$$

Using (5.2), (5.3), the Lebesgue dominated convergence theorem, and the weak convergence $v_{n} \rightarrow v$ in $H^{1}\left(\mathbb{R}^{3}\right)$, for every $z \in C_{0}^{\infty}\left(\mathbb{R}^{3}\right)$, we obtain

$$
\left\langle I^{\prime}\left(v_{n}\right), z\right\rangle \rightarrow\left\langle I^{\prime}(v), z\right\rangle
$$

Since $C_{0}^{\infty}\left(\mathbb{R}^{3}\right)$ is dense in $H^{1}\left(\mathbb{R}^{3}\right)$ and $I^{\prime}\left(v_{n}\right) \rightarrow 0$, we conclude that $I^{\prime}(v)=0$. In other words, we have that $v$ is a critical point of $I$.

Step 2. Now we need to prove that $v \neq 0$. Assume that $v=0$.

By Lemma 4.3 there exist a sequence $\left(y_{n}\right) \subset \mathbb{R}^{3}$ and $r$ and $\eta>0$ such that $\left|y_{n}\right| \rightarrow \infty$ as $n \rightarrow \infty$ and

$$
\lim _{n \rightarrow \infty} \sup \int_{B_{r}\left(y_{n}\right)}\left|v_{n}\right|^{2} d x \geq \eta>0, \quad \forall n \in \mathbb{N} .
$$

Without loss of generality, we may assume that $\left(y_{n}\right) \subset \mathbb{Z}^{3}$. Then defining $u_{n}(x)=v_{n}\left(x+y_{n}\right)$, $n \in \mathbb{N}$, we have $\left\|u_{n}\right\|_{0}=\left\|v_{n}\right\|_{0}$ for all $n \in \mathbb{N}$. Thus, taking a subsequence if necessary, there exists $u \in H^{1}\left(\mathbb{R}^{3}\right)$ such that

$$
\begin{aligned}
& u_{n} \rightarrow u \quad \text { weakly in } H^{1}\left(\mathbb{R}^{3}\right), \\
& u_{n} \rightarrow u \quad \text { strongly in } L_{\text {loc }}^{2}\left(\mathbb{R}^{3}\right), \\
& u_{n}(x) \rightarrow u(x) \quad \text { a.e. on } \mathbb{R}^{3} .
\end{aligned}
$$

From (5.4) we get that $u \neq 0$.

We claim that $u$ is a critical point of $I_{0}$. Indeed, we first observe that

$$
\left\langle I_{0}^{\prime}\left(u_{n}\right), z\right\rangle \rightarrow\left\langle I_{0}^{\prime}(u), z\right\rangle, \quad \forall z \in C_{0}^{\infty}\left(\mathbb{R}^{3}\right),
$$


as $n \rightarrow \infty$. Effectively, writing

$$
\begin{aligned}
& \left\langle I_{0}^{\prime}\left(u_{n}\right), z\right\rangle-\left\langle I_{0}^{\prime}(u), z\right\rangle \\
& \quad=\int_{\mathbb{R}^{3}} \nabla\left(u_{n}-u\right) \nabla z d x+\int_{\mathbb{R}^{3}} V_{0}(x)\left[f\left(u_{n}\right) f^{\prime}\left(u_{n}\right)-f(u) f^{\prime}(u)\right] z d x \\
& \quad+\int_{\mathbb{R}^{3}} K_{0}(x)\left[\widetilde{\phi}_{f\left(u_{n}\right)} f\left(u_{n}\right) f^{\prime}\left(u_{n}\right)-\widetilde{\phi}_{f(u)} f(u) f^{\prime}(u)\right] z d x \\
& \quad-\int_{\mathbb{R}^{3}}\left[g_{0}\left(x, f\left(u_{n}\right)\right)-g_{0}(x, f(u))\right] f^{\prime}\left(u_{n}\right) z d x+o(1) .
\end{aligned}
$$

From the arguments used before we deduce that

$$
\begin{aligned}
& \int_{\mathbb{R}^{3}} \nabla\left(u_{n}-u\right) \nabla z d x \rightarrow 0, \\
& \int_{\mathbb{R}^{3}} V_{0}(x)\left[f\left(u_{n}\right) f^{\prime}\left(u_{n}\right)-f(u) f^{\prime}(u)\right] z d x \rightarrow 0,
\end{aligned}
$$

and

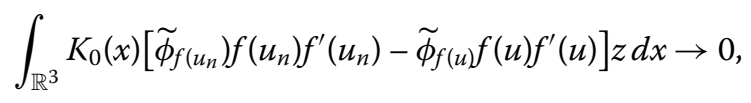

as $n \rightarrow \infty$. Hence, to prove (5.5), it remains to analyze the last integral in (5.6). Note that

$$
\begin{aligned}
{\left[g_{0}\left(x, f\left(u_{n}\right)\right)-g_{0}(x, f(u))\right] f^{\prime}\left(u_{n}\right) z d x=} & {\left[g_{0}\left(x, f\left(u_{n}\right)\right)-g\left(x, f\left(u_{n}\right)\right)\right] f^{\prime}\left(u_{n}\right) z d x } \\
& +\left[g\left(x, f\left(u_{n}\right)\right)-g_{0}(x, f(u))\right] f^{\prime}\left(u_{n}\right) z d x
\end{aligned}
$$

Now, by condition $\left(G_{4}\right)$ (ii) and the arguments used in the proof of (5.3) we get $\widetilde{\psi} \in L^{1}(\Omega)$, $\Omega=\operatorname{supp} z$, such that

$$
\left|\left[g\left(x, f\left(u_{n}\right)\right)-g_{0}(x, f(u))\right] f^{\prime}\left(u_{n}\right) z\right| \leq \widetilde{\psi} .
$$

Hence, applying the Lebesgue dominated convergence theorem once more, we obtain

$$
\left[g\left(x, f\left(u_{n}\right)\right)-g_{0}\left(x, f\left(u_{n}\right)\right)\right] f^{\prime}\left(u_{n}\right) z \rightarrow\left[g(x, f(u))-g_{0}(x, f(u))\right] f^{\prime}(u) z
$$

in $L^{1}(\Omega)$. The claim (5.3) and the Lebesgue dominated convergence theorem also yield

$$
g\left(x, f\left(u_{n}\right)\right) f^{\prime}\left(u_{n}\right) z \rightarrow g(x, f(u)) f^{\prime}(u) z
$$

in $L^{1}(\Omega)$. Furthermore, from (5.3), (5.8), and the Lebesgue dominated convergence theorem we again have

$$
g_{0}(x, f(u)) f^{\prime}\left(u_{n}\right) z \rightarrow g_{0}(x, f(u)) f^{\prime}(u) z
$$

in $L^{1}(\Omega)$. Consequently,

$$
\left[g\left(x, f\left(u_{n}\right)\right)-g_{0}(x, f(u))\right] f^{\prime}\left(u_{n}\right) z \rightarrow\left[g(x, f(u))-g_{0}(x, f(u))\right] f^{\prime}(u) z
$$


in $L^{1}(\Omega)$. Relations (5.7), (5.9), and (5.10) establish the verification of (5.5). On the other hand, considering $z_{n}(x)=z\left(x-y_{n}\right)$ for all $n \in \mathbb{N}$, in view of the periodicities of $V_{0}, K_{0}$, and $g_{0}$, we get

$$
\left\langle I_{0}^{\prime}\left(u_{n}\right), z\right\rangle=\left\langle I_{0}^{\prime}\left(v_{n}\right), z_{n}\right\rangle, \quad \forall n \in \mathbb{N} .
$$

Moreover, applying Lemma 4.4, we have

$$
\left|\left\langle I_{0}^{\prime}\left(v_{n}\right), z_{n}\right\rangle-\left\langle I^{\prime}\left(v_{n}\right), z_{n}\right\rangle\right| \rightarrow 0, \quad \forall n \in \mathbb{N},
$$

as $n \rightarrow \infty$. Since $\left\|z_{n}\right\|_{0}=\|z\|_{0}$ for all $n \in \mathbb{N}$, by (5.11) and (5.12) we conclude that

$$
\left\langle I_{0}^{\prime}\left(v_{n}\right), z_{n}\right\rangle \rightarrow 0
$$

as $n \rightarrow \infty$. This limit, (5.11), and (5.5) show that $u$ is a critical point of $I_{0}$, as claimed.

Step 3.

Claim $I_{0}(u) \leq c$.

To show this fact, we apply the definition of $\left\{u_{n}\right\}$ to get

$$
\begin{aligned}
I\left(v_{n}\right)-\frac{1}{2}\left\langle I^{\prime}\left(v_{n}\right), v_{n}\right\rangle= & \frac{1}{2} \int_{\mathbb{R}^{3}} V_{0}(x)\left[f^{2}\left(u_{n}\right)-f\left(u_{n}\right) f^{\prime}\left(u_{n}\right) u_{n}\right] d x \\
& +\frac{1}{2} \int_{\mathbb{R}^{3}}\left(V(x)-V_{0}(x)\right)\left[f^{2}\left(v_{n}\right)-f\left(v_{n}\right) f^{\prime}\left(v_{n}\right) v_{n}\right] d x \\
& +\frac{1}{4} \int_{\mathbb{R}^{3}} K(x) \phi_{f\left(v_{n}\right)} f^{2}\left(v_{n}\right) d x-\frac{1}{2} \int_{\mathbb{R}^{3}} K(x) \phi_{f\left(v_{n}\right)} f\left(v_{n}\right) f^{\prime}\left(v_{n}\right) v_{n} d x \\
& +\int_{\mathbb{R}^{3}} \frac{1}{2} g\left(x, f\left(v_{n}\right)\right) f^{\prime}\left(v_{n}\right) v_{n}-G\left(x, f\left(v_{n}\right)\right) d x .
\end{aligned}
$$

Now, taking a subsequence if necessary, by property (8) of Lemma 3.1 and Fatou's lemma we obtain

$$
\begin{gathered}
\lim _{n \rightarrow \infty} \inf \frac{1}{2} \int_{\mathbb{R}^{3}} V_{0}(x)\left[f^{2}\left(u_{n}\right)-f\left(u_{n}\right) f^{\prime}\left(u_{n}\right) u_{n}\right] d x \\
\geq \frac{1}{2} \int_{\mathbb{R}^{3}} V_{0}(x)\left[f^{2}(u)-f(u) f^{\prime}(u) u\right] d x .
\end{gathered}
$$

We observe that as $v_{n} \rightarrow 0$ weakly in $H^{1}\left(\mathbb{R}^{3}\right)$, by Lemma 4.4 with $h=V-V_{0}$ and Lemma 3.1(6) we get

$$
\lim _{n \rightarrow \infty} \inf \frac{1}{2} \int_{\mathbb{R}^{3}}\left(V(x)-V_{0}(x)\right)\left[f^{2}\left(v_{n}\right)-f\left(v_{n}\right) f^{\prime}\left(v_{n}\right) v_{n}\right] d x=0 .
$$

We also claim that

$$
\begin{gathered}
\lim _{n \rightarrow \infty} \inf \left[\frac{1}{4} \int_{\mathbb{R}^{3}} K(x) \phi_{f\left(v_{n}\right)} f^{2}\left(v_{n}\right) d x-\frac{1}{2} \int_{\mathbb{R}^{3}} K(x) \phi_{f\left(v_{n}\right)} f\left(v_{n}\right) f^{\prime}\left(v_{n}\right) v_{n} d x\right] \\
\geq \frac{1}{4} \int_{\mathbb{R}^{3}} K_{0}(x) \widetilde{\phi}_{f(u)} f^{2}(u) d x-\frac{1}{2} \int_{\mathbb{R}^{3}} K_{0}(x) \widetilde{\phi}_{f(u)} f(u) f^{\prime}(u) u d x
\end{gathered}
$$


and

$$
\begin{gathered}
\lim _{n \rightarrow \infty} \int_{\mathbb{R}^{3}}\left(\frac{1}{2} g\left(x, f\left(v_{n}\right)\right) f^{\prime}\left(v_{n}\right) v_{n}-G\left(x, f\left(v_{n}\right)\right)\right) d x \\
\geq \int_{\mathbb{R}^{3}}\left(\frac{1}{2} g_{0}(x, f(u)) f^{\prime}(u) u-G_{0}(x, f(u))\right) d x .
\end{gathered}
$$

Assuming that the claims are true, we use (5.1), (5.13) - (5.17), and the fact that $u$ is a critical point of $I_{0}$ to get

$$
\begin{aligned}
c \geq & \frac{1}{2} \int_{\mathbb{R}^{3}} V_{0}(x)\left[f^{2}(u)-f(u) f^{\prime}(u) u\right] d x+\frac{1}{4} \int_{\mathbb{R}^{3}} K_{0}(x) \widetilde{\phi}_{f(u)} f^{2}(u) d x \\
& -\frac{1}{2} \int_{\mathbb{R}^{3}} K_{0}(x) \widetilde{\phi}_{f(u)} f(u) f^{\prime}(u) u d x \\
& +\int_{\mathbb{R}^{3}}\left(\frac{1}{2} g_{0}(x, f(u)) f^{\prime}(u) u-G_{0}(x, f(u))\right) d x \\
= & I_{0}(u)-\frac{1}{2}\left\langle I_{0}^{\prime}(u), u\right\rangle=I_{0}(u),
\end{aligned}
$$

that is, $I_{0}(u) \leq c$.

Step 4. We will verify that $\max _{t \geq 0} I_{0}(t u)=I_{0}(u)$. Define the function $\eta(t):=I_{0}(t u)$ for $t \geq 0$. Since $u$ is a critical point of $I_{0}$, it follows that $u>0$ (see the argument further). Hence by Fubini's theorem we may write

$$
\begin{aligned}
\eta^{\prime}(t)= & t\left\{\int_{\mathbb{R}^{3}}|\nabla u|^{2} d x-\int_{\mathbb{R}^{3}}\left[\frac{g_{0}(x, f(t|u|)) f^{\prime}(t|u|)}{t|u|}\right.\right. \\
& \left.\left.-\frac{V_{0}(x) f(t|u|) f^{\prime}(t|u|)}{t|u|}-\frac{K_{0}(x) \widetilde{\phi}_{f(t|u|)} f(t|u|) f^{\prime}(t|u|)}{t|u|}\right] u^{2} d x\right\} .
\end{aligned}
$$

Note that, for fixed $x \in \mathbb{R}^{3}$, the function $\xi:(0,+\infty \rightarrow \mathbb{R})$ defined by

$$
\xi(s)=\frac{g_{0}(x, f(s)) f^{\prime}(s)}{s}-\frac{V_{0}(x) f(s) f^{\prime}(s)}{s}-\frac{K_{0}(x) \widetilde{\phi}_{f(s)} f(s) f^{\prime}(s)}{s}
$$

is increasing. Indeed, this is a direct consequence of $\left(G_{4}\right)($ iii) and Corollary 3.1(2) applied to

$$
\xi(s)=\frac{g_{0}(x, f(s))}{f^{7}(s)} \frac{f^{3}(s) f^{\prime}(s)}{s} f^{4}(s)+V_{0}(x)\left(-\frac{f(s) f^{\prime}(s)}{s}\right)+K_{0}(x) \widetilde{\phi}_{f(s)}\left(-\frac{f(s) f^{\prime}(s)}{s}\right) .
$$

Now we observe that $\eta^{\prime}(1)=0$ since $u$ is a critical point of $I_{0}$. Moreover, we have that $\eta^{\prime}(t)>0$ for $0<t<1$ and $\eta^{\prime}(t)<0$ for $t>1$. Therefore

$$
I_{0}(u)=\eta(1)=\max _{t \geq 0} \eta(t)=\max _{t \geq 0} I_{0}(t u)
$$

Consequently, by (5.17), $\left(G_{4}\right)(\mathrm{i})$, and the definition of $c$ we have

$$
c \leq \max _{t \geq 0} I(t u) \leq \max _{t \geq 0} I_{0}(t u)=I_{0}(u) \leq c .
$$


This implies that there exists $\gamma \in \Gamma$ such that (2.3) holds. In view of Theorem 2.2, we possess a critical point $v$ on level $c$. From $c \geq \sigma>0=I(0)$ we have that $v$ is a nonzero critical point of $I$. This concludes the proof of Theorem 1.1, except for claims (5.16) and (5.17).

Step 5. We will now show that $v>0$ in $\mathbb{R}^{3}$. Since $v \geq 0$ in $\mathbb{R}^{3}$ is a weak solution of the equation

$$
-\Delta v=w, \quad x \in \mathbb{R}^{3},
$$

where

$$
w=: f^{\prime}(v(x))\left[g(x, f(v(x)))-V(x) f(v(x))-K(x) \phi_{f(v(x)) f} f(v(x))\right],
$$

by conditions $(V),(K),\left(G_{1}\right)$, and $\left(G_{2}\right)$ we have

$$
|w| \leq f^{\prime}(v)\left[\delta|f(v)|+C_{\delta}|f(v)|^{q_{1}-1}\right] \leq C \delta+\widetilde{C}_{\delta}|f(v)|^{\frac{q_{1}-1}{2}}
$$

thanks to Lemma 3.1(2), (7), and (10). Let $p_{0}=\frac{12}{q_{1}-1}>1$. Since $v \in L^{6}\left(\mathbb{R}^{3}\right)$, it follows that $w \in$ $L^{p_{0}}\left(B_{R}\right)$ with arbitrary $R>0$. By elliptic regularity theory, $v \in W^{2, p_{0}}\left(B_{R}\right)$, Using a standard bootstrap argument, we may conclude that $v \in W^{2, p_{0}}\left(B_{R}\right)$ for every $p_{0} \geq 2$. Hence $v \in C_{\text {loc }}^{1, \alpha}$ for some $\alpha \in(0,1)$. Now suppose by contradiction that there is $x_{0} \in \mathbb{R}^{3}$ such that $v\left(x_{0}\right)=0$. Equation (3.4) can be rewritten as

$$
-\Delta v+c(x) v=V(x) f^{\prime}(v)(v-f(v))+K(x) \phi_{f(v)} f^{\prime}(v)(v-f(v))+g(x, f(v)) f^{\prime}(v) \geq 0,
$$

where $c(x)=V(x) f^{\prime}(v)+K(x) \phi_{f(v)} f^{\prime}(v)>0$ for $x \in \mathbb{R}^{3}$ is a continuous function. We also note that from Lemma 3.1(3) we have $v-f(v) \geq 0$. Hence, applying the strong maximum principle for weak solutions (see [22]), we obtain that $v \equiv 0$, which contradicts the fact that $v \not \equiv 0$.

Step 6. Finally, we conclude the proof of Theorem 1.1 by showing that (5.16) and (5.17) hold. Similarly to the proof of Lemma 4.4(3), we have

$$
\int_{\mathbb{R}^{3}} K(x) \phi_{f\left(v_{n}\right)} f\left(v_{n}\right) f^{\prime}\left(v_{n}\right) v_{n} d x \rightarrow \int_{\mathbb{R}^{3}} K_{0}(x) \widetilde{\phi}_{f\left(v_{n}\right)} f\left(v_{n}\right) f^{\prime}\left(v_{n}\right) v_{n} d x
$$

and

$$
\int_{\mathbb{R}^{3}} K(x) \phi_{f\left(v_{n}\right)} f^{2}\left(v_{n}\right) d x \rightarrow \int_{\mathbb{R}^{3}} K_{0}(x) \widetilde{\phi}_{f\left(v_{n}\right)} f^{2}\left(v_{n}\right) d x
$$

strongly in $L^{1}\left(\mathbb{R}^{3}\right)$ as $n \rightarrow \infty$. Consequently, by the periodicity of $K_{0}$,

$$
\begin{aligned}
& \lim _{n \rightarrow \infty} \inf \frac{1}{4} \int_{\mathbb{R}^{3}} K(x) \phi_{f\left(v_{n}\right)} f^{2}\left(v_{n}\right) d x-\frac{1}{2} \int_{\mathbb{R}^{3}} K(x) \phi_{f\left(v_{n}\right)} f\left(v_{n}\right) f^{\prime}\left(v_{n}\right) v_{n} d x \\
& \quad=\lim _{n \rightarrow \infty} \inf \frac{1}{4} \int_{\mathbb{R}^{3}} K_{0}(x) \widetilde{\phi}_{f\left(u_{n}\right)} f^{2}\left(u_{n}\right) d x-\frac{1}{2} \int_{\mathbb{R}^{3}} K_{0}(x) \widetilde{\phi}_{f\left(u_{n}\right)} f\left(u_{n}\right) f^{\prime}\left(u_{n}\right) u_{n} d x
\end{aligned}
$$


Moreover, by Lemma 3.1(6), for $s \geq 0$, we have

$$
\begin{aligned}
& \frac{1}{2} K_{0}(x) \widetilde{\phi}_{f(s)} f^{2}(s)-K_{0}(x) \widetilde{\phi}_{f(s)} f(s) f^{\prime}(s) s \\
&=\frac{1}{2}\left[K_{0}(x) \widetilde{\phi}_{f(s)} f^{2}(s)-K(x) \phi_{f(s)} f^{2}(s)\right]+\frac{1}{2} K(x) \phi_{f(s)} f^{2}(s)-K(x) \phi_{f(s)} f(s) f^{\prime}(s) s \\
& \quad+K(x) \phi_{f(s)} f(s) f^{\prime}(s) s-K_{0}(x) \widetilde{\phi}_{f(s)} f(s) f^{\prime}(s) s \\
& \geq \\
& \geq \frac{1}{2} K(x) \phi_{f(s)} f^{2}(s)+\left[K(x) \phi_{f(s)} f(s) f^{\prime}(s) s-K_{0}(x) \widetilde{\phi}_{f(s)} f(s) f^{\prime}(s) s\right] \\
& \geq-\frac{1}{2} K(x) \phi_{s} s^{2}+\left[K(x) \phi_{f(s)} f^{2}(s)-K_{0}(x) \widetilde{\phi}_{f(s)} f^{2}(s)\right] \\
& \geq-\frac{1}{2} K(x) \phi_{s} s^{2}-\left[K_{0}(x) \widetilde{\phi}_{s} s^{2}-K(x) \phi_{s} s^{2}\right] .
\end{aligned}
$$

Claim $\int_{\mathbb{R}^{3}} K(x) \phi_{u_{n}} u_{n}^{2} d x \rightarrow \int_{\mathbb{R}^{3}} K(x) \phi_{u} u^{2} d x$.

Proof By the result in [7], if $u_{n} \rightarrow u$ in $H^{1}\left(\mathbb{R}^{3}\right)$, then we have $u_{n}^{2} \rightarrow u^{2}$ in $L^{3}\left(\mathbb{R}^{3}\right)$ and $\phi_{u_{n}} \rightarrow$ $\phi_{u}$ in $D^{1,2}\left(\mathbb{R}^{3}\right)$, so that $\phi_{u_{n}} \rightarrow \phi_{u}$ in $L^{6}\left(\mathbb{R}^{3}\right)$. Therefore

$$
\begin{aligned}
\int_{\mathbb{R}^{3}} K(x) \phi_{u_{n}} u_{n}^{2} d x-K(x) \phi_{u} u^{2} d x= & \int_{\mathbb{R}^{3}} K(x) \phi_{u_{n}}\left(u_{n}^{2}-u^{2}\right) d x \\
& +\int_{\mathbb{R}^{3}} K(x)\left(\phi_{u_{n}}-\phi_{u}\right) u^{2} d x
\end{aligned}
$$

Since

$$
\int_{\mathbb{R}^{3}}\left(K(x) \phi_{u_{n}}\right)^{\frac{3}{2}} d x \leq|K|_{2}^{\frac{3}{2}}\left|\phi_{u_{n}}\right|_{6}^{\frac{3}{2}}<+\infty,
$$

so that $K(x) \phi_{u_{n}} \in L^{\frac{3}{2}}\left(\mathbb{R}^{3}\right)$, we have $\int_{\mathbb{R}^{3}} K(x) \phi_{u_{n}}\left(u_{n}^{2}-u^{2}\right) d x \rightarrow 0$. Similarly,

$$
\int_{\mathbb{R}^{3}}\left(K(x) u^{2}\right)^{\frac{6}{5}} d x \leq|K|_{2}^{\frac{6}{5}}|u|_{6}^{\frac{12}{5}}<+\infty
$$

so that $K(x) u^{2} \in L^{\frac{6}{5}}\left(\mathbb{R}^{3}\right)$, and thus $\int_{\mathbb{R}^{3}} K(x)\left(\phi_{u_{n}}-\phi_{u}\right) u^{2} d x \rightarrow 0$.

We obtain $\int_{\mathbb{R}^{3}} K_{0}(x) \widetilde{\phi}_{u_{n}} u_{n}^{2} d x \rightarrow \int_{\mathbb{R}^{3}} K_{0}(x) \widetilde{\phi}_{u} u^{2} d x$ in the same way. Thus by Fatou's lemma we have

$$
\begin{aligned}
\lim _{n \rightarrow \infty} & \inf \int_{\mathbb{R}^{3}}\left[\frac{1}{4} K_{0}(x) \widetilde{\phi}_{f\left(u_{n}\right)} f^{2}\left(u_{n}\right)-\frac{1}{2} K_{0}(x) \widetilde{\phi}_{f\left(u_{n}\right)} f\left(u_{n}\right) f^{\prime}\left(u_{n}\right) u_{n}\right. \\
& \left.+\frac{1}{2} K(x) \phi_{u_{n}} u_{n}^{2}+K_{0}(x) \widetilde{\phi}_{u_{n}} u_{n}^{2}-K(x) \phi_{u_{n}} u_{n}^{2}\right] d x \\
\geq & \int_{\mathbb{R}^{3}}\left[\frac{1}{4} K_{0}(x) \widetilde{\phi}_{f(u)} f^{2}(u)-\frac{1}{2} K_{0}(x) \widetilde{\phi}_{f(u)} f(u) f^{\prime}(u) u\right. \\
& \left.+\frac{1}{2} K(x) \phi_{u} u^{2}+K_{0}(x) \widetilde{\phi}_{u} u^{2}-K(x) \phi_{u} u^{2}\right] d x
\end{aligned}
$$

Relations (5.19) and (5.20) conclude the verification of (5.16). The proof of (5.17) can be found in [34]. The proof of Theorem 1.1 is completed. 
Proof of Theorem 1.2 We argue as in the initial steps of the proof of Theorem 1.1. Since $g_{0}$ satisfies $\left(G_{1}\right),\left(G_{2}\right)$, and $(G)$, applying Corollary 4.1 , we can find a sequence $\left\{v_{n}\right\} \subset H^{1}\left(\mathbb{R}^{3}\right)$ such that

$$
I_{0}\left(v_{n}\right) \rightarrow c \geq \sigma>0, \quad\left\|I_{0}^{\prime}\left(v_{n}\right)\right\|\left(1+\left\|v_{n}\right\|\right) \rightarrow 0 \quad \text { as } n \rightarrow \infty,
$$

where $c$ is given by Theorem 2.1. Because of Lemma 4.2, we may suppose, without loss of generality, that $v_{n} \rightarrow v$ weakly in $H^{1}\left(\mathbb{R}^{3}\right)$. From this and (3.6) we have that $v$ is a critical point of $I_{0}$, that is, $I_{0}^{\prime}(v)=0$. To finish the proof of the theorem, it suffices to assume that $v=0$.

By Lemma 4.3 there exist a sequence $\left(y_{n}\right) \subset \mathbb{R}^{3}$ and $r, \eta>0$ such that $\left|y_{n}\right| \rightarrow \infty$ as $n \rightarrow \infty$ and

$$
\lim _{n \rightarrow \infty} \sup \int_{B_{r}\left(y_{n}\right)}\left|v_{n}\right|^{2} d x \geq \eta>0, \quad \forall n \in \mathbb{N} .
$$

Without loss of generality we may assume that $\left(y_{n}\right) \subset \mathbb{Z}$. Defining $u_{n}(x)=v_{n}\left(x+y_{n}\right), n \in \mathbb{N}$, we have that $\left\|u_{n}\right\|_{0}=\left\|v_{n}\right\|_{0}$ for all $n \in \mathbb{N}$. Consequently, taking a subsequence if necessary, there exists $u \in H^{1}\left(\mathbb{R}^{3}\right)$ such that $u_{n} \rightarrow u$ weakly in $H^{1}\left(\mathbb{R}^{3}\right), u_{n} \rightarrow u$ strongly in $L_{\text {loc }}^{2}\left(\mathbb{R}^{3}\right)$, and $u_{n}(x) \rightarrow u(x)$ almost everywhere on $\mathbb{R}^{3}$. We claim that $u$ is a critical point of $I_{0}$. Indeed, given $w \in H^{1}\left(\mathbb{R}^{3}\right)$, from $(V),(K),\left(G_{1}\right)$, and $\left(G_{2}\right)$ we get

$$
\left\langle I_{0}^{\prime}\left(u_{n}\right), w\right\rangle \rightarrow\left\langle I_{0}^{\prime}(u), w\right\rangle \quad \text { as } n \rightarrow \infty .
$$

On the other hand, considering $w_{n}(x)=w\left(x-y_{n}\right)$ for all $n \in \mathbb{N}$, in view of the periodicities of $V_{0}, K_{0}$, and $g_{0}$, we get

$$
\left\langle I_{0}^{\prime}\left(u_{n}\right), w\right\rangle \rightarrow\left\langle I_{0}^{\prime}\left(v_{n}\right), w_{n}\right\rangle, \quad n \in \mathbb{N}
$$

Consequently, from (5.21) and the fact that $\left\|w_{n}\right\|_{0}=\|w\|_{0}$ for all $n \in \mathbb{N}$ we conclude that

$$
\left\langle I_{0}^{\prime}\left(u_{n}\right), w\right\rangle \rightarrow 0 \quad \text { as } n \rightarrow \infty .
$$

This limit, together with (5.23), shows that $u$ is a critical point of $I_{0}$. The claim is proved. Furthermore, (5.22) implies that $u \neq 0$. Theorem 1.2 is proved.

\footnotetext{
Acknowledgements

The authors would like to thank the referees for valuable comments and suggestions for this manuscript.

Funding

JZ was supported by the Natural Science Foundation of Inner Mongolia Autonomous Region (No. 2019MS01004) and the National Natural Science Foundation of China (No. 11962025). MY was supported by the Natural Science Foundation of Shandong Province (No. ZR2019PA020).
}

Availability of data and materials

We declare that materials described in the manuscript are freely available to any scientist wishing to use them for noncommercial purposes, without breaching participant confidentiality. 
Authors' contributions

The authors declare that the study was realized in collaboration with the same responsibility. All authors read and approved the final manuscript.

\section{Author details}

${ }^{1}$ Mathematics Sciences College, Inner Mongolia Normal University, Hohhot, P.R. China. ${ }^{2}$ School of Mathematics and Statistics, Northeast Petroleum University, Daqing, P.R. China. ${ }^{3}$ School of Mathematics and Statistics, Qilu University of Technology (Shandong Academy of Sciences), Jinan, P.R. China.

\section{Publisher's Note}

Springer Nature remains neutral with regard to jurisdictional claims in published maps and institutional affiliations.

Received: 15 April 2020 Accepted: 31 May 2020 Published online: 16 June 2020

\section{References}

1. Alves, C.O., do Ó, J.M., Miyagaki, O.H.: On nonlinear perturbations of a periodic in $\mathbb{R}^{2}$ involving critical growth. Nonlinear Anal. 56, 781-791 (2004)

2. Alves, C.O., Souto, M.A.S., Soares, S.H.M.: Schrödinger-Poisson equations without Ambrosetti-Rabinowitz condition J. Math. Anal. Appl. 377, 584-592 (2011)

3. Ambrosetti, A., Rabinowitz, P.H.: Dual variational methods in critical point theory and applications. J. Funct. Anal. 14 349-381 (1973)

4. Azzollini, A., Pomponio, A.: Ground state solutions for the nonlinear Schrödinger-Maxwell equations. J. Math. Anal. Appl. 345, 90-108 (2008)

5. Benmilh, K., Kavian, O.: Existence and asymptotic behaviour of standing waves for quasilinear Schrödinger-Poisson systems in $\mathbb{R}^{3}$. Ann. Inst. Henri Poincaré, Anal. Non Linéaire 25, 449-470 (2008)

6. Berestycki, H., Lions, P.L.: Nonlinear scalar field equations, l: existence of a ground state. Arch. Ration. Mech. Anal. 82, 313-346 (1982)

7. Cerami, G., Vaira, G.: Positive solutions for some non-autonomous Schrödinger-Poisson systems. J. Differ. Equ. 248, 521-543 (2010)

8. Chen, L.Z., Feng, X.J., Hao, X.N.: The existence of sign-changing solution for a class of quasilinear Schrödinger-Poisson systems via perturbation method. Bound. Value Probl. 2019, Article ID 159 (2019)

9. Chen, S.T., Tang, X.H.: On the planar Schrödinger-Poisson system with the axially symmetric potential. J. Differ. Equ. 268, 945-976 (2020)

10. Colin, G.M.: A multiplicity result for nonlinear Schrödinger-Maxwell equation. Commun. Appl. Anal. 7, 417-423 (2003)

11. Colin, G.M., Jeanjean, L.: Solutions for a quasilinear Schrödinger equation: a dual approach. Nonlinear Anal. 56 213-226 (2004)

12. D’Ambrosio, L., Mitidieri, E.: Quasilinear elliptic systems in divergence form associated to general nonlinearities. Adv. Nonlinear Anal. 7, 425-447 (2018)

13. D'Aprile, T., Mugnai, D.: Solitary waves for nonlinear Klein-Gordon-Maxwell and Schrödinger-Maxwell equations. Proc. R. Soc. Edinb., Sect. A 134, 893-906 (2004)

14. D'Aprile, T., Wei, J.C.: On bound states concentrating on sphere for the Maxwell-Schrödinger equations. SIAM J. Math. Anal. 15, 321-342 (2005)

15. Ding, L., Lin, L., Meng, Y.J., Zhuang, G.L.: Existence and asymptotic behavior of ground state for quasilinear Schrödinger-Poisson system in $\mathbb{R}^{3}$. Topol. Methods Nonlinear Anal. 47, 241-264 (2016)

16. do Ó, J.M., Severo, U.B.: Quasilinear Schrödinger equations involving concave and convex nonlinearities. Commun. Pure Appl. Anal. 8, 621-644 (2009)

17. do Ó, J.M., Severo, U.B.: Solitary waves for a class of quasilinear Schrödinger equations in dimension two. Calc. Var. Partial Differ. Equ. 38, 275-315 (2010)

18. Fang, X.D., Szulkin, A.: Multiple solutions for a quasilinear Schrödinger equation. J. Differ. Equ. 254, $2015-2032$ (2013)

19. Figueiredo, G.M., Siciliano, G.: Existence and asymptotic behaviour of solutions for a quasi-linear Schrödinger-Poisson system under a critical nonlinearity (2017). arXiv:1707.05353

20. Figueiredo, G.M., Siciliano, G.: Quasi-linear Schrödinger-Poisson system under an exponential critical nonlinearity: existence and asymptotic behaviour of solutions. Arch. Math. 112, 313-327 (2019)

21. Fiscella, A.: A fractional Kirchhoff problem involving a singular term and a critical nonlinearity. Adv. Nonlinear Anal. 8, 645-660 (2019)

22. Gilbarg, D., Trudinger, N.S.: Elliptic Partial Differential Equations of Second Order. Springer, Berlin (1983)

23. Ianni, I.: Solutions of the Schrödinger-Poisson problem concentrating on spheres, part II: existence. Math. Models Methods Appl. Sci. 19, 877-910 (2009)

24. Ianni, I., Varia, G.: Solutions of the Schrödinger-Poisson problem concentrating on spheres, part I: necessary condition. Math. Models Methods Appl. Sci. 19, 707-720 (2009)

25. Illner, R., Kavian, O., Lange, H.: Stationary solutions of quasi-linear Schrödinger-Poisson system. J. Differ. Equ. 145, $1-16(1998)$

26. Jabri, Y.: The Mountain Pass Theorem—Variants, Generalizations and Some Applications. Encyclopedia of Mathematics and Its Applications. Cambridge University Press, Cambridge (2003)

27. Ji, C., Fang, F., Zhang, B.L.: Least energy sign-changing solutions for the nonlinear Schrödinger-Poisson system. Electron. J. Differ. Equ. 2017, Article ID 282 (2017)

28. Ji, C., Fang, F., Zhang, B.L.: A multiplicity result for asymptotically linear Kirchhoff equations. Adv. Nonlinear Anal. 8 , 267-277 (2019)

29. Li, W., Rădulescu, V., Zhang, B.L.: Infinitely many solutions for fractional Kirchhoff-Schrödinger-Poisson systems. J. Math. Phys. 60, Article ID 011506 (2019) 
30. Liu, J.Q., Wang, L.Q., Wang, Z.-Q.: Soliton solutions for quasilinear Schrödinger equations II. J. Differ. Equ. 187, 473-493 (2003)

31. Mao, A.M., Chang, H.J.: Schrödinger-Poisson system with radial potentials and discontinuous quasilinear nonlinearity. Topol. Methods Nonlinear Anal. 51, 79-89 (2018)

32. Mingqi, X., Rădulescu, V.D., Zhang, B.L.: A critical fractional Choquard-Kirchhoff problem with magnetic field. Commun. Contemp. Math. 21, Article ID 185004 (2019)

33. Shen, L.J: Ground state solutions for a class of generalized quasilinear Schrödinger-Poisson systems. Bound. Value Probl. 2018, Article ID 44 (2018)

34. Silva, E.A.B., Vieira, G.F.: Quasilinear asymptotically periodic Schrödinger equations with subcritical growth. Nonlinear Anal. 72, 2935-2949 (2010)

35. Tang, X.: Non-Nehari manifold method for asymptotically periodic Schrödinger equations. Sci. China Math. 58, 715-728 (2015)

36. Tang, X., Lin, X.Y., Yu, J.S.: Existence of a bound state solution for quasilinear Schrödinger equations. J. Dyn. Differ. Equ. 31, 369-383 (2019)

37. Willem, M.: Minimax Theorems. Birkhäuser, Boston (1996)

38. Xiang, M., Wang, F.: Fractional Schrödinger-Poisson-Kirchhoff type systems involving critical nonlinearities. Nonlinear Anal. 164, 1-26 (2017)

39. Xiang, M., Zhang, B.L., Yang, D.: Multiplicity results for variable-order fractional Laplacian equations with variable growth. Nonlinear Anal. 178, 190-204 (2019)

40. Xiang, M.Q., Rădulescu, V.D., Zhang, B.L.: Fractional Kirchhoff problems with critical Trudinger-Moser nonlinearity. Calc. Var. Partial Differ. Equ. 58, Article ID 57 (2019)

41. Xiang, M.Q., Rădulescu, V.D., Zhang, B.L.: Nonlocal Kirchhoff problems with singular exponential nonlinearity. Appl. Math. Optim. (2020). https://doi.org/10.1007/s00245-020-09666-3

42. Xiang, M.Q., Zhang, B.L., Rădulescu, V.D.: Superlinear Schrödinger-Kirchhoff type problems involving the fractional p-Laplacian and critical exponent. Adv. Nonlinear Anal. 9, 690-709 (2020)

43. Xue, Y.F., Tang, C.L.: Existence of a bound state solution for quasilinear Schrödinger equations. Adv. Nonlinear Anal. 8, 323-338 (2019)

44. Ye, Y.W., Tang, C.L.: Existence and multiplicity of solutions for Schrödinger-Poisson equations with sign-changing potential. Calc. Var. Partial Differ. Equ. 53, 383-411 (2015)

45. Zhang, H., Xu, J.X., Zhang, F.B.: Positive ground states for asymptotically periodic Schrödinger-Poisson systems. Math. Methods Appl. Sci. 36, 427-439 (2013)

46. Zhang, H., Xu, J.X., Zhang, F.B., Du, M.: Ground states for asymptotically periodic Schrödinger-Poisson systems with critical growth. Cent. Eur. J. Math. 12, 1484-1499 (2014)

47. Zhao, L., Liu, H.D., Zhao, F.K.: Existence and concentration of solutions for the Schrödinger-Poisson equations with steep well potential. J. Differ. Equ. 255, 1-23 (2013)

\section{Submit your manuscript to a SpringerOpen ${ }^{\circ}$ journal and benefit from:}

- Convenient online submission

- Rigorous peer review

- Open access: articles freely available online

- High visibility within the field

- Retaining the copyright to your article

Submit your next manuscript at $\boldsymbol{\nabla}$ springeropen.com 